\title{
Long-range behavior of valence nucleons in a hyperspherical formalism
}

\author{
N. K. Timofeyuk \\ Physics Department, University of Surrey, Guildford, Surrey GU2 7XH, England, United Kingdom
}

(Received 19 January 2007; published 11 October 2007)

\begin{abstract}
It is shown that hyperspherical harmonics can be represented in a form typical of traditional microscopic cluster models. This allows those hyperharmonics that are responsible for long-range behavior of valence nucleons in loosely bound nuclei to be selected. The hyperspherical cluster model based on such hyperharmonics is tested for ${ }^{5} \mathrm{He}$ in a "toy model" that uses a simplified description of the ${ }^{4} \mathrm{He}$ core. The comparison of the hyperspherical expansion to a microscopic cluster model calculation confirms the feasibility of the hyperspherical treatment of long-range behavior by basis convergence only.
\end{abstract}

DOI: 10.1103/PhysRevC.76.044309

PACS number(s): 21.60.-n, 27.10.+h

\section{INTRODUCTION}

A knowledge of the wave functions at large distances gives information about various nuclear reactions. An ability to calculate wave functions in external regions makes different applications possible, such as the prediction of low-energy reaction rates for astrophysics. On the other hand, the structure quantities that characterize nuclear wave functions at large distances (such as phase shifts and asymptotic normalization coefficients) depend on the choice of nucleon-nucleon $(N N)$ interaction. Therefore, comparison between predicted and observed values gives an opportunity to study the $N N$ interactions in more detail. The long-range behavior is especially important for loosely bound nuclei with a large excess of neutrons or protons.

It is not easy nor always possible to obtain correct long-range behavior from first principles. It can be obtained naturally only for three-body and four-body systems using Faddeev and Faddeev-Yakubovsky equations. For threenucleon systems, correct long-range behavior has also been achieved using pair correlated hyperspherical harmonics [1], which has allowed asymptotic normalization constants for the $n-d$ and $p$ - $d$ systems to be calculated. The hyperspherical formalism has also been applied to calculations of the overlap integral between triton and ${ }^{4} \mathrm{He}$ [2]. Despite the very large basis space used in these calculations, the proper asymptotic behavior of the overlap $\left\langle\left. t \otimes p\right|^{4} \mathrm{He}\right\rangle$, given by the Whittaker function with relevant proton separation energy, has not been achieved at $r>6 \mathrm{fm}$. To restore this behavior, the authors solved the inhomogeneous equation for overlap integrals from Ref. [3]. For heavier nuclei, only a few ab initio calculations of overlap integrals are available. Large-scale no-core shell model calculations of the overlap $\left\langle\left.{ }^{7} \mathrm{Be} \otimes p\right|^{8} \mathrm{~B}\right\rangle[4]$ have not provided the required decrease at $r>5 \mathrm{fm}$ so that the authors had to match this overlap to the Whittaker function, or approximate it by solutions of a Woods-Saxon potential to use this overlap in reaction calculations. Three other ab initio overlap calculations are available within the variational Monte-Carlo method, for $\left\langle{ }^{4} \mathrm{He} \otimes d \mid{ }^{6} \mathrm{Li}\right\rangle[5],\left\langle{ }^{4} \mathrm{He} \otimes t \mid{ }^{7} \mathrm{Li}\right\rangle$, and $\left\langle{ }^{4} \mathrm{He} \otimes{ }^{3} \mathrm{He} \mid{ }^{7} \mathrm{Be}\right\rangle[6]$. The radial behavior of the long-range part of these overlaps is imposed there prior to calculations so that it is not determined by the differences in calculated energies of the nucleus and the clusters that compose it.
Many-body approaches that use Slater determinants made of single-particle wave functions have also to adjust the potential wells to fit the experimental energies for valence nucleons if such wave functions are to be used in reaction calculations. However, this procedure does not necessarily provide the separation energy that is the difference between the total energies. Also, it may be inconsistent with $N N$ interactions employed in these approaches.

Proper long-range behavior can be guaranteed if the total wave function $\Psi$ is represented by an antisymmetried product of the core $\phi_{c}$ and valence $\phi_{\text {val }}$ wave functions,

$$
\Psi=\mathcal{A}\left(\phi_{c} \otimes \phi_{\mathrm{val}}\right)
$$

and then the microscopic $R$-matrix approach is used determine the function $\phi_{\text {val }}$ [7]. At present a microscopic cluster model (MCM) of this type is used to predict cross sections of astrophysically relevant low-energy reactions [8]. However, this model is only able to deal with oscillator shell model core wave functions $\phi_{c}$ and can not be used with realistic $N N$ interactions that reproduce $N N$ data. Although in this model the separation energies are always equal to the differences of the total binding energies, they often differ significantly from experimental values so that one parameter of the effective $N N$ potential should be tuned.

The problem of the long-range behavior can be resolved within the hyperspherical interpolation approach proposed in Ref. [9]. This approach has been designed to describe manynucleon systems near thresholds when identified channels having a binary cluster structure are believed to play an important role, especially in the nuclear surface and beyond. In this approach the wave function of a many-nucleon system $\Psi$ is represented by two terms $\Psi=\Psi_{1}+\Psi_{2}$, one of which explicitly contains the binary channel wave function. Both $\Psi_{1}$ and $\Psi_{2}$ are expanded in a hyperspherical basis and a system of equations that couples them derived by substituting the total wave function $\Psi$ into the general expression for the variational principle. The interpolation approach does not involve matching at some surface. The coupling between the two components of the wave function is governed by the $N N$ interaction through the Schrödinger equation. Earlier applications of the interpolation method included the calculation of ${ }^{4} \mathrm{He}+n$ elastic scattering [10]. Later, this approach 
was used to study the long-range behavior in ${ }^{8} \mathrm{~B}$ and ${ }^{6} \mathrm{Li}$ nuclei within a three-body model [11]. Similar ideas have been successfully used to describe the ${ }^{3} \mathrm{He}+p$ scattering within the hyperspherical formalism [12]. The success of these approaches suggests that the hyperspherical interpolation method can be applied to heavier systems as well. However, to make it applicable to other systems, a hyperspherical expansion that contains contributions only from the long-range part of the wave function $\Psi$ should be introduced.

This article is a new step toward extending the interpolation approach beyond the three- and four-body systems. It introduces a subset of hyperspherical basis that is expected to be responsible for long-range behavior. A new method to calculate matrix elements in such a basis is developed. In the future, this basis can be used to solve different problems that require explicit treatment of wave functions in asymptotic regions. However, prior to any advanced applications, the new method must be tested for a simple case. In this article the applicability of the proposed approach is illustrated by the example of the "toy model" of "He that uses a simplified description of the ${ }^{4} \mathrm{He}$ core. The results for such a case should be very close to those obtained with a traditional microscopic cluster model. The comparison between the hyperspherical expansion and the MCM presented in this article for artificially bound ${ }^{5} \mathrm{He}$ confirms the feasibility of the hyperspherical treatment of long-range behavior by basis convergence only.

In Sec. II the hyperspherical cluster basis is constructed and in Sec. III the expansion onto this basis is discussed. The link between the hyperspherical cluster model and MCM is shown in Sec. IV, while the representation of hyperspherical cluster harmonics by oscillator cluster wave functions is given in Sec. V. A method to calculate matrix elements in this basis is presented in Sec. VI and the application to ${ }^{5} \mathrm{He}$ is discussed in Sec. VII. The results obtained are summarized in Sec. VIII. Finaly, exact formulas for the norm and the two-body $N N$ potential matrix elements as well as for the overlap integral are derived in the Appendix.

\section{HYPERSPHERICAL CLUSTER HARMONICS}

A nucleus $A$ can be described in hyperspherical coordinates, which include the hyperradius $\rho$,

$$
\rho^{2}=\sum_{i=1}^{A-1} \xi_{i}^{2}
$$

where $\boldsymbol{\xi}_{i}$ are the normalized Jacobi coordinates,

$$
\boldsymbol{\xi}_{i}=\sqrt{\frac{i}{i+1}}\left(\frac{1}{i} \sum_{j=1}^{i} \boldsymbol{r}_{j}-\boldsymbol{r}_{i+1}\right)
$$

and a set of $3 A-4$ hyperangles $\hat{\rho}$. The wave function $\Psi$ written in these coordinates can be expanded into hyperspherical harmonics $(\mathrm{HHs})$ that depend on the hyperangles.

Let us consider a nucleus $A$ that is strongly clusterized as a core $A-1$ plus a weakly bound valence nucleon $N$. It is well-known [13] that the spatial HHs for $A$ nucleons can be written as a product of a spatial HHs for $A-1$ nucleons, the spherical function $Y_{l m}\left(\hat{\boldsymbol{\xi}}_{A-1}\right)$, and a function $\varphi_{n}(\theta)$, which is an eigenfunction of the angular part of Laplacian in variable $\theta$ defined as $\theta=\arctan \xi_{A-1} / \rho_{c}$. Here $\rho_{c}$ is the hyperradius for the $A-1$ core, $\rho^{2}=\rho_{c}^{2}+\xi_{A-1}^{2}$. The angle $\theta$ is associated with the valence nucleon and it shows how far the valence nucleon is for a fixed size of the core $A-1$. To describe the long-range behavior of the last nucleon for a fixed structure of the core, it is necessary to include as many eigenfunction $\varphi_{n}(\theta)$ as possible.

This article accounts for cluster structure $(A-1)+N$ of weakly bound nuclei by reorganizing known recurrence representation of HHs in a form that is similar to the microscopic cluster model. This is achieved by antisymmetrizing the product of a (known) completely antisymmetric $\mathrm{HH}$, $Y_{K_{c} \gamma_{c}}^{M_{L_{c}} M_{S_{c}} M_{T_{c}}}\left(\hat{\boldsymbol{\rho}}_{c}\right)$, for the core $A-1$ (spin-isospin part included) and a relative angular function, $\varphi_{K_{c} n l m \sigma \tau}\left(\theta, \hat{\xi}_{A-1}\right)$,

$$
\begin{aligned}
\mathcal{Y}_{K_{c} \gamma_{c} n l L S T}^{M_{L} M_{S} M_{T}}(\hat{\boldsymbol{\rho}})= & \mathcal{N}_{K_{c} \gamma_{c} n l L S T}^{-1} \sum_{M_{L_{c}} M_{S_{c}} M_{T_{c}} m \sigma \tau}\left(L_{c} M_{L_{c}} l m \mid L M_{L}\right) \\
& \times\left(S_{c} M_{S_{c}} \frac{1}{2} \sigma \mid S M_{S}\right)\left(T_{c} M_{T_{c}} \frac{1}{2} \tau \mid T M_{T}\right) \\
& \times \mathcal{A}\left(Y_{K_{c} \gamma_{c}}^{M_{L_{c}} M_{S_{c}} M_{T_{c}}}\left(\hat{\boldsymbol{\rho}}_{c}\right) \varphi_{K_{c} n l m \sigma \tau}\left(\theta, \hat{\xi}_{A-1}\right)\right) .
\end{aligned}
$$

Here $K_{c}$ is the hypermoment; $\gamma_{c}=\left\{\beta_{c} L_{c} S_{c} T_{c}\right\} ; L_{c}, S_{c}$, and $T_{c}$ are the total orbital momentum, spin, and isospin of the core $A-1$, and $\beta_{c}$ represents different degenerate harmonics. Also,

$$
\mathcal{A}=\frac{1}{A^{1 / 2}}\left(1-\sum_{i=1}^{A-1} P_{i A}\right)
$$

is the antisymmetrization operator that permutes the $A$-th nucleon with nucleons of the core, $\mathcal{N}_{K_{c} \gamma_{c} n l L S T}$ is a normalization factor, $\hat{\boldsymbol{\rho}}_{c}$ denotes the hyperangles for the $A-1$-body system, and

$$
\begin{aligned}
\varphi_{K_{c} n l m \sigma \tau}\left(\theta, \hat{\boldsymbol{\xi}}_{A-1}\right)= & N_{n K_{c} l}(\sin \theta)^{l}(\cos \theta)^{K_{c}} \\
& \times P_{n}^{l+1 / 2, K_{c}+(3 A-8) / 2}(\cos 2 \theta) \\
& \times Y_{l m}\left(\hat{\boldsymbol{\xi}}_{A-1}\right) \chi_{\sigma \tau}(A),
\end{aligned}
$$

where $\quad \cos \theta=\rho_{c} / \rho, \quad \sin \theta=\xi_{A-1} / \rho, \quad \cos 2 \theta=1-$ $2 \xi_{A-1}^{2} / \rho^{2}, \chi_{\sigma \tau}(A)$ is the spin-isospin function of the $A$-th nucleon with the spin and isospin projections $\sigma$ and $\tau$, respectively, and

$$
N_{n K_{c} l}^{2}=\frac{2 n !\left(2 n+K_{c}+l+(3 A-5) / 2\right) \Gamma\left(n+K_{c}+l+(3 A-5) / 2\right)}{\Gamma(n+l+3 / 2) \Gamma\left(n+K_{c}+(3 A-6) / 2\right)} .
$$


Because $Y_{K_{c} \gamma_{c}}^{M_{L_{c}} M_{S_{c}} M_{T_{c}}}\left(\hat{\boldsymbol{\rho}}_{c}\right)$ and $\varphi_{K_{c} n l m \sigma \tau}\left(\theta, \hat{\boldsymbol{\xi}}_{A-1}\right)$ are the eigenfunctions of the operator of the kinetic energy in subspaces associated with variables $\hat{\boldsymbol{\rho}}_{c}$ and $\left\{\hat{\boldsymbol{\xi}}_{A-1}, \theta\right\}$ and because the operator of the kinetic energy is symmetric and the function (4) is antisymmetric with respect to any nucleon permutations, the function $\mathcal{Y}_{K_{c} \gamma_{c} n l L S T}^{M_{L} M_{T}}(\hat{\boldsymbol{\rho}}) \equiv \mathcal{Y}_{K \gamma}(\hat{\boldsymbol{\rho}})$ is also an eigenfunction of the $3 A-4$-dimensional angular part $\Delta_{\hat{\rho}}$ of the Laplacian,

$$
\Delta_{\hat{\rho}} \mathcal{Y}_{K \gamma}(\hat{\boldsymbol{\rho}})=-K(K+3 A-5) \mathcal{Y}_{K \gamma}(\hat{\rho}),
$$

and therefore it is the $\mathrm{HH}$ with the hypermoment $K=K_{c}+$ $2 n+l$. Here $\gamma=\left\{K_{c} \gamma_{c} n l L S T M_{L} M_{S} M_{T}\right\}$.

Because the HHs $\mathcal{Y}_{K \gamma}(\hat{\boldsymbol{\rho}})$ are written in the form typical for the cluster wave functions (1), they are referred to below as hyperspherical cluster harmonics $(\mathrm{HCH})$. The $\mathrm{HCHs} \mathcal{Y}_{K \gamma}(\hat{\boldsymbol{\rho}})$ with different values of $K$ are orthogonal to each other; however, for the same $K$ and different $\gamma$ they may be not orthogonal,

$$
\left\langle\mathcal{Y}_{K^{\prime} \gamma^{\prime}}(\hat{\boldsymbol{\rho}}) \mid \mathcal{Y}_{K \gamma}(\hat{\boldsymbol{\rho}})\right\rangle=\delta_{K K^{\prime}} \mathcal{I}_{K \gamma \gamma^{\prime}},
$$

which is common for different channel functions in multichannel cluster models with antisymmetrization.

\section{EXPANSION OF NUCLEAR WAVE FUNCTIONS ONTO HYPERSPHERICAL CLUSTER BASIS}

The wave function of a nucleus with one weakly bound nucleon can be expanded onto the hyperspherical cluster basis,

$$
\Psi=\rho^{-(3 A-4) / 2} \sum_{K \gamma} \chi_{K \gamma}(\rho) \mathcal{Y}_{K \gamma}(\hat{\boldsymbol{\rho}}),
$$

keeping only a restricted number of quantum numbers in the $\mathrm{HHs}$ of the core and as much relative functions (6) (or in other words as many $n^{\prime}$ s) as needed to describe properly the long-range radial behavior of the valence nucleon. However, when more then one set of quantum numbers for the $\mathrm{HH}$ of the core is present in the expansion (10), the hyperspherical cluster basis may not be orthogonal for the same $K$. The simplest way to deal with this problem is to introduce the orthogonalized HHs,

$$
\tilde{\mathcal{Y}}_{K \tilde{\gamma}}(\hat{\boldsymbol{\rho}})=\sum_{\gamma} A_{\gamma \tilde{\gamma}} \mathcal{Y}_{K \gamma}(\hat{\boldsymbol{\rho}}),
$$

which can be achieved, for example, by performing the singular value decomposition of the matrix $\mathcal{I}_{K \gamma \gamma^{\prime}}$. Then the decomposition

$$
\Psi=\rho^{-(3 A-4) / 2} \sum_{K \tilde{\gamma}} \tilde{\chi}_{K \tilde{\gamma}}(\rho) \tilde{\mathcal{Y}}_{K \tilde{\gamma}}(\hat{\boldsymbol{\rho}})
$$

onto the orthogonal basis $\tilde{\mathcal{Y}}_{K \tilde{\gamma}}(\hat{\boldsymbol{\rho}})$ leads to a standard set of differential hyperradial equations of the Hyperspherical Function Method (HSFM),

$$
\begin{aligned}
& \left(\frac{d^{2}}{d \rho^{2}}-\frac{\mathcal{L}_{K}\left(\mathcal{L}_{K}+1\right)}{\rho^{2}}-\frac{2 m}{\hbar^{2}}\left(E+\tilde{V}_{K \tilde{\gamma}, K \tilde{\gamma}}(\rho)\right)\right) \tilde{\chi}_{K \tilde{\gamma}}(\rho) \\
& \quad=\frac{2 m}{\hbar^{2}} \sum_{K^{\prime} \tilde{\gamma}^{\prime} \neq K \tilde{\gamma}} \tilde{V}_{K \tilde{\gamma}, K^{\prime} \tilde{\gamma}^{\prime}}(\rho) \tilde{\chi}_{K^{\prime} \tilde{\gamma}^{\prime}}(\rho),
\end{aligned}
$$

where $\mathcal{L}_{K}=K+(3 A-6) / 2, m$ is the nucleon mass, and the hyperradial potentials $\tilde{V}_{K \tilde{\gamma}, K^{\prime} \tilde{\gamma}^{\prime}}(\rho)$ are the matrix elements of the $N N$ interactions

$$
\tilde{V}_{K \tilde{\gamma}, K^{\prime} \tilde{\gamma}^{\prime}}(\rho)=\left\langle\tilde{\mathcal{Y}}_{K \tilde{\gamma}}(\hat{\boldsymbol{\rho}})\left|\sum_{i<j} V_{i j}\left(\boldsymbol{r}_{i}-\boldsymbol{r}_{j}\right)\right| \tilde{\mathcal{Y}}_{K^{\prime} \tilde{\gamma}^{\prime}}(\hat{\boldsymbol{\rho}})\right\rangle .
$$

The functions $\chi_{K \gamma}(\rho)$ corresponding to the nonorthogonal $\mathrm{HCH}$ basis $\mathcal{Y}_{K \gamma}(\hat{\boldsymbol{\rho}})$ with well-defined quantum numbers $\left\{K_{c} \gamma_{c} n l\right\}$ are then obtained using the transformation

$$
\chi_{K \gamma}(\rho)=\sum_{\tilde{\gamma}} A_{\gamma \tilde{\gamma}} \tilde{\chi}_{K \tilde{\gamma}}(\rho) .
$$

\section{LINK BETWEEN THE HYPERSPHERICAL CLUSTER MODEL AND THE MICROSCOPIC CLUSTER MODEL}

If the expansion (10) retains only a limited number of $\left\{K_{c} \gamma_{c}\right\}$ while at each fixed $\left\{K_{c} \gamma_{c}\right\}$ the summation over all quantum numbers $n$ is performed, such an expansion corresponds to a specific cluster model of the type shown in Eq. (1). The easiest way to demonstrate this is to retain only one state $\left\{K_{c} \gamma_{c}\right\}$ for the core $A-1$ and neglect all spin couplings. Then the wave function (10) can be rewritten as

$$
\begin{aligned}
\Psi= & \mathcal{A}\left(\sum_{n} \mathcal{N}_{K_{c} \gamma_{c} n l L S T}^{-1} \frac{\chi_{K_{c} \gamma_{c} n l}(\rho)}{\rho^{(3 A-4) / 2}} Y_{k_{c} \gamma_{c}}\left(\hat{\boldsymbol{\rho}}_{c}\right)\right. \\
& \left.\times \varphi_{K_{c} n l m \sigma \tau}\left(\theta, \hat{\boldsymbol{\xi}}_{A-1}\right)\right) .
\end{aligned}
$$

If after the antisymmetrization operator in Eq. (16) a closure relation is inserted,

$$
\sum_{i}\left|\Psi_{K_{c} \gamma_{c}}^{(i)}\left(\rho_{c}, \hat{\boldsymbol{\rho}}_{c}\right)\right\rangle\left\langle\Psi_{K_{c} \gamma_{c}}^{(i)}\left(\rho_{c}, \hat{\boldsymbol{\rho}}_{c}\right)\right|=1,
$$

where $\Psi_{K_{c} \gamma_{c}}^{(i)}\left(\rho_{c}, \hat{\boldsymbol{\rho}}_{c}\right)$ is the $i$-th solution of the Schrödinger equation for $A-1$ nucleons in the hyperspherical basis that keeps only one selected $\mathrm{HH}, Y_{k_{c} \gamma_{c}}\left(\hat{\boldsymbol{\rho}}_{c}\right)$, then the wave function (10) can be rewritten as

$$
\Psi=\sum_{i} \mathcal{A}\left(\Psi_{K_{c} \gamma_{c}}^{(i)}\left(\rho_{c}, \hat{\boldsymbol{\rho}}_{c}\right) \phi_{K_{c} \gamma_{c}}^{(i)}\left(\boldsymbol{\xi}_{A-1}\right)\right),
$$

with the relative function $\phi_{K_{c} \gamma_{c}}^{(i)}\left(\boldsymbol{\xi}_{A-1}\right)$ determined by the expression

$$
\begin{aligned}
\phi_{K_{c} \gamma_{c}}^{(i)}\left(\boldsymbol{\xi}_{A-1}\right)= & \sum_{n} \mathcal{N}_{K_{c} \gamma_{c} n l L S T}^{-1}\left\langle\phi_{K_{c} \gamma_{c}}^{(i)}\left(\rho_{c}, \hat{\boldsymbol{\rho}}_{c}\right) Y_{k_{c} \gamma_{c}}\left(\hat{\boldsymbol{\rho}}_{c}\right)\right| \\
& \left.\times \frac{\chi_{K \gamma}\left(\sqrt{\rho_{c}+\xi_{A-1}^{2}}\right)}{\left(\rho_{c}^{2}+\xi_{A-1}^{2}\right)^{(3 A-4) / 4}} \varphi_{K_{c} n l m \sigma \tau}\left(\boldsymbol{\xi}_{A-1}\right)\right\rangle
\end{aligned}
$$

Thus the $\mathrm{HCH}$ expansion of the wave function $\Psi$ is equivalent to a microscopic multichannel cluster model that includes all the excited states of the core $A-1$ that correspond to the hyperradial excitations of the lowest state with $\left\{K_{c} \gamma_{c}\right\}$.

In the general case, when more sets of the quantum numbers $\left\{K_{c} \gamma_{c}\right\}$ are retained in the $\mathrm{HCH}$ expansion, it is possible to 
show that the $\mathrm{HCH}$ expansion corresponds to the multichannel cluster model that includes all the excited states of the core with total spin $J_{c}$, which can be constructed with the values of $L_{c}$ and $S_{c}$ retained in the core description, and their hyperradial excitations. Such a derivation is quite cumbersome and is not given here.

\section{LINK BETWEEN HYPERSPHERICAL CLUSTER HARMONICS AND OSCILLATOR CLUSTER WAVE FUNCTIONS}

The $\mathrm{HCH} \mathcal{Y}_{K \gamma}(\hat{\rho})$ can be represented by the cluster wave functions that contain oscillator shell model wave functions. Such a representation is used below to develop a technique to calculated the matrix elements in the $\mathrm{HCH}$ basis. The link to the cluster shell model can be derived using an expansion of the Jacobi polynomials into the radial oscillator wave functions $R_{\kappa l}(\xi)$ [see Eqs. (A1)-(A3) in the Appendix] and representing the core $\mathrm{HH} Y_{K_{c} \gamma_{c}}\left(\hat{\boldsymbol{\rho}}_{c}\right)$ as

$$
Y_{K_{c} \gamma_{c}}\left(\hat{\boldsymbol{\rho}}_{c}\right)=\Psi_{0 K_{c} \gamma_{c}}\left(\boldsymbol{\xi}_{1}, \ldots, \boldsymbol{\xi}_{A-2}\right) / R_{0 K_{c}}\left(\rho_{c}\right)
$$

where $\Psi_{0 K_{c} \gamma_{c}}\left(\boldsymbol{\xi}_{1}, \ldots, \boldsymbol{\xi}_{A-2}\right)$ is the translation-invariant oscillator shell model function without the hyperradial excitations,

$$
R_{0 K_{c}}\left(\rho_{c}\right)=b^{-(3 A-6) / 2} \sqrt{\frac{2}{\Gamma\left(K_{c}+(3 A-6) / 2\right)}}\left(\frac{\rho_{c}}{b}\right)^{K_{c}} e^{-\rho_{c}^{2} / 2 b^{2}}
$$

is the hyperradial oscillator wave function, and $b$ is an arbitrary oscillator radius. For the lowest possible value of $K_{c}$ the $\Psi_{0 K_{c} \gamma_{c}}\left(\boldsymbol{\xi}_{1}, \ldots, \boldsymbol{\xi}_{A-2}\right)$ is the usual $0 \hbar \omega$ translationinvariant shell model wave function. For higher $K_{c}$, $\Psi_{0 K_{c} \gamma_{c}}\left(\xi_{1}, \ldots, \boldsymbol{\xi}_{A-2}\right)$ can be constructed, for example, using the technique of Ref. [14]. With Eqs. (21), (20), and (A1)-(A3) one gets

$$
\mathcal{Y}_{K \gamma}(\hat{\boldsymbol{\rho}})=\mathcal{N}_{K \gamma}^{-1} \sum_{\nu=0}^{n} \sum_{\kappa=0}^{\nu} B_{n K_{c} l}^{\nu \kappa} Z_{\alpha \nu}(\hat{\boldsymbol{\rho}}),
$$

where $\alpha \equiv\left\{K_{c} \gamma_{c} \kappa l L S T M_{L} M_{S} M_{T}\right\}$,

$$
\begin{aligned}
Z_{\alpha v}(\hat{\boldsymbol{\rho}})= & b^{K_{c}+2 v+l+(3 A-3) / 2} \rho^{-K_{c}-l-2 v} e^{\rho^{2} / 2 b^{2}} \\
& \times \mathcal{A} \Phi_{\alpha}^{(b)}\left(\boldsymbol{\xi}_{1}, \ldots \boldsymbol{\xi}_{A-1}\right),
\end{aligned}
$$

and $\Phi_{\alpha}^{(b)}\left(\xi_{1}, \ldots \xi_{A-1}\right)$ is a non-antisymmetrized oscillator cluster wave function,

$$
\begin{aligned}
\Phi_{\alpha}^{(b)}\left(\boldsymbol{\xi}_{1}, \ldots \boldsymbol{\xi}_{A-1}\right)= & \sum_{M_{L_{c}} M_{S_{c}} M_{T_{c}} m \sigma \tau}\left(L_{c} M_{L_{c}} \operatorname{lm} \mid L M_{L}\right) \\
& \times\left(S_{c} M_{S_{c}} \frac{1}{2} \sigma \mid S M_{S}\right)\left(T_{c} M_{T_{c}} \frac{1}{2} \tau \mid T M_{T}\right) \\
& \times \Psi_{0 K_{c} \gamma_{c}}^{M_{L_{c}} M_{S_{c}} M_{T_{c}}}\left(\boldsymbol{\xi}_{1}, \ldots, \boldsymbol{\xi}_{A-2}\right) \\
& \times R_{\kappa l m}\left(\boldsymbol{\xi}_{A-1}\right) \chi_{\sigma \tau}(A),
\end{aligned}
$$

corresponding to the oscillator radius $b$. The expansion coefficients $B_{n K_{c} l}^{\nu \kappa}$ are given by the expression

$$
\begin{aligned}
B_{n K_{c} l}^{\nu \kappa}= & (-)^{\nu+\kappa} \nu ! N_{n K_{c} l} \\
& \times\left(\frac{\Gamma\left(K_{c}+(3 A-6) / 2\right) \Gamma(\kappa+l+3 / 2)}{4 \kappa !}\right)^{1 / 2} \\
& \times\left(\begin{array}{c}
v+l+1 / 2 \\
\kappa+l+1 / 2
\end{array}\right)\left(\begin{array}{c}
n+l+1 / 2 \\
n-v
\end{array}\right) \\
& \times\left(\begin{array}{c}
n+K_{c}+l+(3 A-7) / 2+v \\
n+K_{c}+l+(3 A-7) / 2
\end{array}\right)
\end{aligned}
$$

\section{MATRIX ELEMENTS IN THE HYPERSPHERICAL CLUSTER BASIS}

The matrix elements in the hyperspherical cluster basis for an arbitrary operator $\hat{O}$ can be calculated using the link (22) between the $\mathrm{HCH}$ and the oscillator cluster wave function. This gives

$$
\begin{aligned}
\left\langle\mathcal{Y}_{K^{\prime} \gamma^{\prime}}(\hat{\boldsymbol{\rho}})|\hat{O}| \mathcal{Y}_{K \gamma}(\hat{\boldsymbol{\rho}})\right\rangle= & \left(\mathcal{N}_{K^{\prime} \gamma^{\prime}} \mathcal{N}_{K \gamma}\right)^{-1} \sum_{\nu=0}^{n} \sum_{v^{\prime}=0}^{n^{\prime}} \sum_{\kappa=0}^{v} \sum_{\kappa^{\prime}=0}^{v^{\prime}} \\
& \times B_{n^{\prime} K_{c}^{\nu^{\prime} l^{\prime}}} B_{n K_{c} l}^{\nu \kappa}\left\langle Z_{\alpha^{\prime} \nu^{\prime}}(\hat{\boldsymbol{\rho}})|\hat{O}| Z_{\alpha \nu}(\hat{\boldsymbol{\rho}})\right\rangle .
\end{aligned}
$$

The matrix elements $\left\langle Z_{\alpha^{\prime} v^{\prime}}(\hat{\boldsymbol{\rho}})|\hat{O}| Z_{\alpha \nu}(\hat{\boldsymbol{\rho}})\right\rangle$ can be calculated using a technique described in Ref. [14] that replaces the integration over hyperangles by the Laplace transform of the shell model matrix elements. This results in

$$
\begin{aligned}
& \left\langle Z_{\alpha^{\prime} \nu^{\prime}}(\hat{\boldsymbol{\rho}})|\hat{O}| Z_{\alpha v}(\hat{\boldsymbol{\rho}})\right\rangle \\
& =\rho^{-K_{c}-K_{c}^{\prime}-2\left(v+v^{\prime}\right)-l-l^{\prime}-3 A+5} \frac{1}{\pi i} \\
& \quad \times \int_{-i \infty}^{i \infty} d s e^{s \rho^{2}} s^{-\left(K_{c}+K_{c}^{\prime}+l+l^{\prime}+3 A-3\right) / 2-v-v^{\prime}} O_{\alpha^{\prime}}^{\alpha}\left(s^{-1 / 2}\right),
\end{aligned}
$$

where

$$
\begin{aligned}
O_{\alpha^{\prime}}^{\alpha}(b)= & \int d \xi_{1} \cdots d \xi_{A-1} \mathcal{A}\left(\Phi_{\alpha^{\prime}}^{(b)}\left(\xi_{1}, \ldots \xi_{A-1}\right)\right) \\
& \times \hat{O} \mathcal{A}\left(\Phi_{\alpha}^{(b)}\left(\xi_{1}, \ldots \xi_{A-1}\right)\right)
\end{aligned}
$$

are the translation-invariant cluster shell model matrix elements corresponding to the oscillator radius $b=s^{-2}$.

\section{A. Normalization of hyperspherical cluster harmonics}

The normalization coefficients $\mathcal{N}_{K \gamma}$ of the $\mathrm{HCH}$ are obtained using Eqs. (9) and (26)-(28) with $\hat{O}=1$. Because the norm $I_{\alpha \alpha^{\prime}}^{S M}=\left\langle\mathcal{A} \Phi_{\alpha^{\prime}} \mid \mathcal{A} \Phi_{\alpha}\right\rangle$ of the oscillator shell model cluster functions does not depend on $b$ the integration over $s$ 
in Eq. (27) can be performed immediately. This gives

$$
\begin{aligned}
\mathcal{I}_{K \gamma \gamma^{\prime}}= & \frac{2}{\mathcal{N}_{K \gamma} \mathcal{N}_{K \gamma^{\prime}}} \sum_{\nu=0}^{n} \sum_{\nu^{\prime}=0}^{n} \sum_{\kappa=0}^{\nu} \sum_{\kappa^{\prime}=0}^{v^{\prime}} \\
& \times \frac{\delta_{K_{c}^{\prime}+2 \kappa^{\prime}+l^{\prime}, K_{c}+2 \kappa+l} B_{n K_{c}^{\prime} \nu^{\prime}}^{\nu^{\prime}} B_{n K_{c} l}^{\nu \kappa} I_{\alpha \alpha^{\prime}}^{S M}}{\Gamma\left(\frac{K_{c}+K_{c}^{\prime}+l+l^{\prime}+3 A-3}{2}+v+v^{\prime}\right)} .
\end{aligned}
$$

Then $\mathcal{N}_{K \gamma}$ is found from the condition $\mathcal{I}_{K \gamma \gamma}=1$.

It is useful here to clarify the meaning of this normalization coefficient. Let us introduce a fractional parentage expansion of the HCH (4) onto the complete set of HHs $Y_{K_{c} \gamma_{c}}\left(\hat{\boldsymbol{\rho}}_{c}\right)$ for the $A-1$ nucleons:

$$
\begin{aligned}
\mathcal{Y}_{K \gamma}(\hat{\boldsymbol{\rho}})= & \sum_{K_{c} \gamma_{c} M_{L_{c}} M_{S_{c}} M_{T_{c}} m \sigma \tau}\left\langle A K \gamma \mid A-1 K_{c} \gamma_{c}, l\right\rangle \\
& \times Y_{K_{c} \gamma_{c}}\left(\hat{\boldsymbol{\rho}}_{c}\right) \varphi_{K_{c} n l m \sigma \tau}\left(\theta, \hat{\boldsymbol{\xi}}_{A-1}\right)\left(L_{c} M_{L_{c}} l m \mid L M_{L}\right) \\
& \times\left(S_{c} M_{S_{c}} \frac{1}{2} \sigma \mid S M_{S}\right)\left(T_{c} M_{T_{c}} \frac{1}{2} \tau \mid T M_{T}\right),
\end{aligned}
$$

where $\left\langle A K \gamma \mid A-1 K_{c} \gamma_{c}, l\right\rangle$ is the fractional expansion coefficient. Multiplying Eq. (30) by $Y_{K_{c} \gamma_{c}}\left(\hat{\boldsymbol{\rho}}_{c}\right), \varphi_{K_{c} n l m \sigma \tau}\left(\theta, \hat{\boldsymbol{\xi}}_{A-1}\right)$, and three relevant Clebsch-Gordan coefficients and then integrating over angular variables and making summations over all projections of angular momenta and spins, we get

$$
\mathcal{N}_{K \gamma}=\sqrt{A}\left\langle A K \gamma \mid A-1 K_{c} \gamma_{c}, l\right\rangle .
$$

Thus, the normalization coefficient $\mathcal{N}_{K \gamma}$ is directly related to the fractional expansion coefficient into the core state on which the $\mathrm{HCH}$ is originally built. The knowledge of this relation is very useful for calculating the overlap integrals between the wave functions of nuclei $A$ and $A-1$.

The overlap $I_{\alpha \alpha^{\prime}}^{S M}$ that enters Eq. (29) can be calculated using the fact that for an arbitrary function, $\psi_{\alpha}=\phi_{\alpha_{1}}(1, \ldots$, $A-1) \varphi_{\alpha_{2}}(A)$, where $\phi$ is an antisymmetric function,

$$
\begin{aligned}
\left\langle\mathcal{A} \psi_{\alpha^{\prime}} \mid \mathcal{A} \psi_{\alpha}\right\rangle= & A^{1 / 2}\left\langle\phi_{\alpha_{1}^{\prime}}(1, \ldots, A-1) \varphi_{\alpha_{2}^{\prime}}(A) \mid \mathcal{A} \psi_{\alpha}\right\rangle \\
= & \delta_{\alpha \alpha^{\prime}}-(A-1)\left\langle\phi_{\alpha_{1}^{\prime}}(1, \ldots, A-1) \varphi_{\alpha_{2}^{\prime}}(A)\right| \\
& \left.\times \phi_{\alpha_{1}}(1, \ldots, A-2, A) \varphi_{\alpha_{2}}(A-1)\right\rangle, \quad
\end{aligned}
$$

where $\mathcal{A}$ is determined by Eq. (5). The exchange term in this expression can be calculated using the fractional parentage expansion of the translation-invariant oscillator wave function of $A-1$ nucleons. The details of these calculations and final analytical expressions are given in Sec. B of the Appendix.

\section{B. Matrix elements of central two-body $N \boldsymbol{N}$ interactions}

In this article, only central two-body $N N$ forces are considered,

$$
V=\sum_{i<j}^{A} \sum_{s, t=0,1} V_{i j}^{(s t)}\left(\left|\boldsymbol{r}_{i}-\boldsymbol{r}_{j}\right|\right) \hat{P}_{s t}(i, j) .
$$

Here $\hat{P}_{s t}(i, j)$ is the projector into states with spin $s$ and isospin $t$ of the pair of nucleons $(i, j)$. To calculate the matrix elements of this potential in the $\mathrm{HCH}$ basis using Eq. (26), one needs to know the matrix elements $V_{\alpha^{\prime} \alpha}^{S M}$ in the oscillator shell model cluster basis, given by Eq. (28) with $\hat{O}=V$. The latter can be found using the fact that for an arbitrary function $\psi_{\alpha}=\phi_{\alpha_{1}}(1, \ldots, A-1) \varphi_{\alpha_{2}}(A)$ that contains a product of antisymmetric function $\phi_{i}$

$$
\begin{aligned}
\left\langle\mathcal{A} \psi_{\alpha^{\prime}}|V| \mathcal{A} \psi_{\alpha}\right\rangle= & A^{1 / 2}\left\langle\phi_{\alpha_{1}^{\prime}}(1, \ldots, A-1) \varphi_{\alpha_{2}^{\prime}}(A)|V| \mathcal{A} \psi_{\alpha}\right\rangle \\
= & \left\langle\phi_{\alpha_{1}^{\prime}}(1, \ldots, A-1) \varphi_{\alpha_{2}^{\prime}}(A)|V|\right. \\
& \left.\times \phi_{\alpha_{1}}(1, \ldots, A-1) \varphi_{\alpha_{2}}(A)\right\rangle-(A-1) \\
& \times\left\langle\phi_{\alpha_{1}^{\prime}}(1, \ldots, A-1) \varphi_{\alpha_{2}^{\prime}}(A)|V|\right. \\
& \left.\times \phi_{\alpha_{1}}(1, \ldots, A-2, A) \varphi_{\alpha_{2}}(A-1)\right\rangle .
\end{aligned}
$$

Eq. (34) contains direct and exchange terms that can be evaluated with the fractional parentage expansion technique. The derivation of formulas for $V_{\alpha^{\prime} \alpha}^{S M}$ is given in Sec. C of the Appendix and the final expression for the potential $V_{\alpha \alpha^{\prime}}^{S M}$ is

$$
V_{\alpha \alpha^{\prime}}^{S M}=\sum_{n_{0}^{\prime} n_{0} l_{0} s t} \frac{1-(-)^{l_{0}+s+t}}{2} \mathcal{C}_{n_{0}^{\prime} n_{0} l_{0} s t}^{\kappa \kappa^{\prime}}\left\langle\psi_{n_{0}^{\prime} l_{0}}\left\|V^{s t}\right\| \psi_{n_{0} l_{0}}\right\rangle,
$$

where

$$
\left\langle\psi_{n_{0}^{\prime} l_{0}}\left\|V^{s t}\right\| \psi_{n_{0} l_{0}}\right\rangle=\int_{0}^{\infty} d r r^{2} \psi_{n_{0}^{\prime} l_{0}}(r) V^{s t}(\sqrt{2} r) \psi_{n_{0} l_{0}}(r)
$$

is the matrix element of the $N N$ potential between the two-body oscillator wave functions $\psi_{n_{0} l_{0}}(r)$. The analytical expressions for coefficients $\mathcal{C}_{n_{0}^{\prime} 0_{0} l_{0} s}^{\kappa \kappa^{\prime}}$ are given in the Sec. C of the Appendix.

Finally, the hyperradial potentials $V_{K \gamma, K^{\prime} \gamma^{\prime}}(\rho)$ are calculated by using Eq. (26) and applying the Laplace transform (27) to the shell model $N N$ matrix elements $V_{\alpha \alpha^{\prime}}^{S M}$. This gives

$$
V_{K \gamma, K^{\prime} \gamma^{\prime}}(\rho)=\left(\mathcal{N}_{K^{\prime} \gamma^{\prime}} \mathcal{N}_{K \gamma}\right)^{-1} \sum_{n_{0}^{\prime} n_{0} l_{0} s t} \sum_{\nu \nu^{\prime}} v_{n_{0}^{\prime} n_{0} l_{0} s t}^{v v^{\prime}}(\rho) w_{n_{0}^{\prime} n_{0} l_{0} s t}^{\gamma \gamma^{\prime} v v^{\prime}},
$$

where

$$
\begin{aligned}
v_{n_{0}^{\prime} n_{0} l_{0} s t}^{v v^{\prime}}(\rho)= & \rho^{-K_{c}-K_{c}^{\prime}-2\left(v+v^{\prime}\right)-l-l^{\prime}-3 A+5} \frac{1}{2 \pi i} \\
& \times \int_{-i \infty}^{i \infty} d s e^{s \rho^{2}} s^{-\left(K_{c}+K_{c}^{\prime}+l+l^{\prime}+3 A-3\right) / 2-\nu-v^{\prime}} \\
& \times\left\langle\psi_{n_{0}^{\prime} l_{0}}\left\|V^{s t}\right\| \psi_{n_{0} l_{0}}\right\rangle
\end{aligned}
$$

and

$$
w_{n_{0}^{\prime} n_{0} l_{0} s t}^{\gamma \gamma^{\prime} \nu v^{\prime}}=2 \sum_{\kappa=0}^{\nu} \sum_{\kappa^{\prime}=0}^{\nu^{\prime}} \mathcal{C}_{n_{0}^{\prime} n_{0} l_{0} s t}^{\kappa \kappa^{\prime}} B_{n^{\prime} K_{c}^{\prime} l^{\prime}}^{\nu^{\prime} \prime^{\prime}} B_{n K_{c}}^{\nu \kappa}
$$

\section{APPLICATION TO ${ }^{5} \mathrm{He}$}

The hyperspherical cluster model (HCM) proposed here is tested for the simplest cluster nucleus, ${ }^{5} \mathrm{He}={ }^{4} \mathrm{He}+n$. The real ${ }^{5} \mathrm{He}$ is unbound. This means that boundary conditions must be implemented into the Schrödinger equation for a correct description of this system. Without this, the HCM expansion cannot converge and in the limit of infinite basis the ${ }^{5} \mathrm{He}$ energy should approach the ${ }^{4} \mathrm{He}+n$ threshold and the radius tend to infinity. Therefore, keeping in mind future applications for heavier loosely bound nuclei, we artificially 
bind ${ }^{5} \mathrm{He}$ by changing the $N N$ interation in odd partial waves, for which converged results can be expected.

In the present study, only one, the lowest order, hyperspherical harmonics, $Y_{000}\left(\hat{\rho}_{c}\right)$, is retained in the $\mathrm{HCH}$ basis states to describe the core ${ }^{4} \mathrm{He}$ so that the chosen hyperspherical cluster basis

$$
\mathcal{Y}_{K \gamma}(\hat{\boldsymbol{\rho}})=\mathcal{N}_{K \gamma}^{-1} \mathcal{A}\left(Y_{000}\left(\hat{\boldsymbol{\rho}}_{c}\right) \varphi_{K_{c} n l m \sigma \tau}\left(\theta, \hat{\boldsymbol{\xi}}_{A-1}\right)\right),
$$

where $n=(K-l) / 2$, is orthogonal. The norm of this $\mathrm{HCH}$ and the coefficients $\mathcal{C}_{n_{0}^{\prime} n_{0} l_{0} s t}^{\kappa \alpha^{\prime}}$ required for calculations of the hyperradial potentials are derived using the shell model technique from Sec. C of the Appendix that gives

$$
I_{\kappa \kappa^{\prime}}^{S M}=\delta_{\kappa \kappa^{\prime}}\left(1+4^{-2 \kappa-1}\right)
$$

and

$$
\begin{aligned}
\mathcal{C}_{n_{0}^{\prime} n_{0} l_{0} s t}^{\kappa \kappa^{\prime}}= & 3\left(1+2^{-4 \kappa-3}\right) \delta_{\kappa \kappa^{\prime}} \delta_{n_{0}, 0} \delta_{n_{0}^{\prime}, 0} \delta_{l_{0}, 0} \\
& -3 \delta_{l_{0}, 0}\left(\delta_{n_{0}, 0} T_{0,0,0, \kappa 1,00 \kappa^{\prime} 1,1}^{0000,00 n_{n}^{\prime} 0}+\delta_{n_{0}^{\prime}, 0} T_{0,0,0, \kappa^{\prime} 1,00 \kappa 1,1}^{0000,00 n_{0} 0}\right. \\
& +\frac{(2 s+1)(2 t+1)}{2} \sum_{N \Lambda} \\
& \times\left\langle 00 \kappa 1: 1\left|\frac{3}{5}\right| n_{0} l_{0} N \Lambda: 1\right\rangle\left\langle 00 \kappa^{\prime} 1: 1\left|\frac{3}{5}\right| n_{0}^{\prime} l_{0} N \Lambda: 1\right\rangle .
\end{aligned}
$$

To solve the hyperradial coupled equations, the computer code STURMXX [15] was used.

The calculations are performed with the Volkov V1 potential [16] neglecting the Coulomb interaction. Three values of the Majorana parameter $m$ are used, the standard value $m=0.6$, which gives unbound ${ }^{5} \mathrm{He}$, and two nonstandard ones, $m=0.3$ and $m=0.4$, which make the $N N$ interaction in odd partial waves attractive. As shown below, with $m=0.3$, ${ }^{5} \mathrm{He}$ is bound and the neutron separation energy is relatively small, about $2 \mathrm{MeV}$. For $m=0.4$, this separation energy is even smaller, about $140 \mathrm{keV}$. Such a case is interesting as a "toy model" representing halo nuclei. The resulting binding energies and rms radii for all the values of $m$ used are shown in Figs. 1(a) and 1(b) as a function of the cutoff hypermoment $K_{\max }=2 n_{\max }+1$. The binding energy and the rms radius of the core ${ }^{4} \mathrm{He}$, calculated in the lowest approximation of the HSFM, are shown in these figures by horizontal lines (these values do not depend on $m$ ). For $K_{\max } \leqslant 11$, the HCM results are compared to those obtained in the HSFM calculations using the full set of HHs for each $K$ and the technique from Ref. [14].

As expected, for $m=0.6$, the ${ }^{5} \mathrm{He}$ binding energy slowly decreases with $K_{\max }$ while its rms radius diverges. However, for $m=0.3$, convergence of the binding energy and the rms radius is excellent. Because of the simplistic ${ }^{4} \mathrm{He}$ cluster structure, this binding energy converges to an energy that is higher than the energy achieved by the full HSFM calculations, as expected. The full HSFM energy is either $-33.3 \mathrm{MeV}$, if obtained by exponential extrapolation of the HSFM energies at $1 \leqslant K_{\max } \leqslant 11$, or $-33.8 \mathrm{MeV}$ if only the three last points are used to make the extrapolation. The converged HCM energy is $-30.66 \mathrm{MeV}$. The missing $3 \mathrm{MeV}$ comes from neglecting all possible excitations of the ${ }^{4} \mathrm{He}$ core. For $m=0.4$, the five-body $\mathrm{HCM}$ binding energy, obtained in the same model space, can be considered as converged because for $K_{\max }=23$ it differs from the extrapolated value, $-28.72 \mathrm{MeV}$, only by $60 \mathrm{keV}$. However, because this energy is too close to the ${ }^{4} \mathrm{He}+n$ threshold of $-28.57 \mathrm{MeV}$, the convergence in relative energy is not yet achieved. A few more basis states are needed. The difference in total energies between the HCM and the extrapotaled full HSFM five-body energies is $1.97 \mathrm{MeV}$, which is smaller than in the $m=0.3$ case.

It has been shown in Ref. [17] that clustering leads to a particular symmetry in the total wave function. According to Ref. [17], if each of two clusters have a fixed internal symmetry with respect to the kinematic rotation group $O_{A-1}$, the expansion of the total wave function should contain only those $\mathrm{HHs}$ that correspond to the irreducible representations (irreps) $\left(\lambda_{1}+2 i, \lambda_{2}, \lambda_{3}\right)$ of the orthogonal group $O_{A-1}$, where $i=0,1,2, \ldots, \infty$. For ${ }^{5} \mathrm{He}$, the HSFM energy calculated in Ref. [18] with V1 and $m=0.6$ using only irreps $\lambda=$
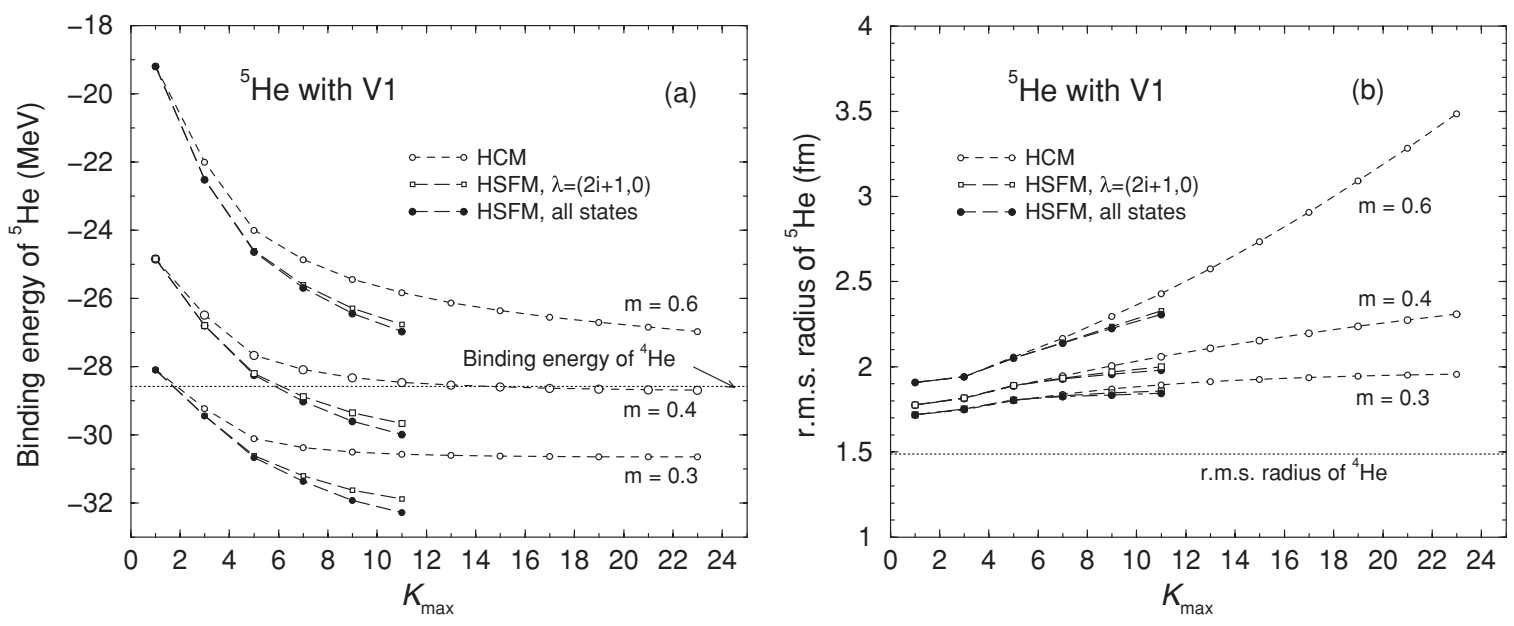

FIG. 1. Binding energy (a) and the rms radius (b) of ${ }^{5} \mathrm{He}$ calculated in the HCM for different cutoff hypermoments $K_{\max }$. The HSFM calculations, performed in a restricted basis that contains only irreps $\lambda=(2 i+1,0)$, where $i=0,1, \ldots,\left(K_{\max }-1\right) / 2$ (open squares), and in full basis (filled circles), are shown as well. The binding energy and the rms radius of the ${ }^{4} \mathrm{He}$ core are represented by dotted horizontal lines. 

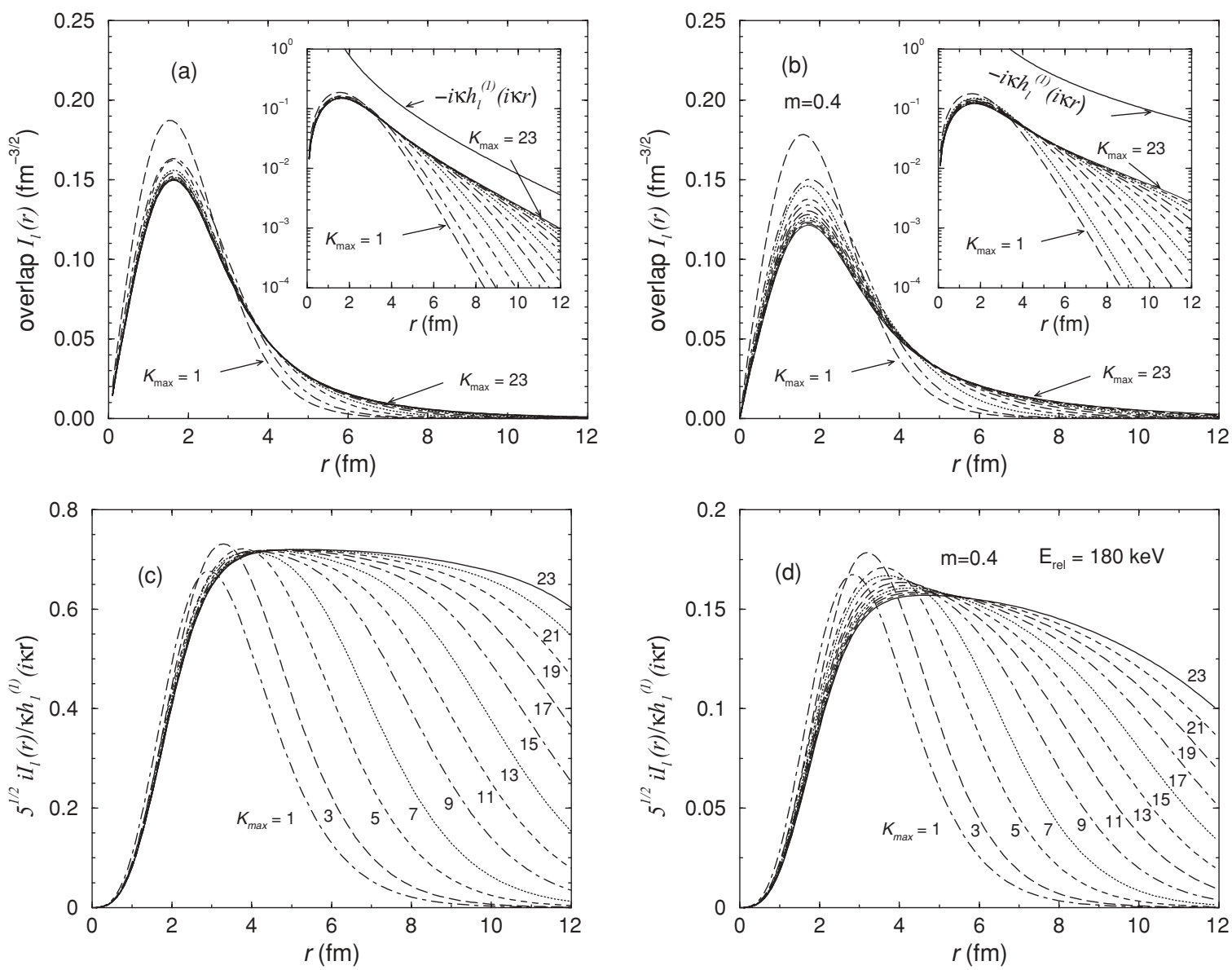

FIG. 2. Overlap integrals (a,b) for ${ }^{5} \mathrm{He}$, shown in linear (main graph) and logarithmic (inset) scales, and their ratios to the function $-i \kappa h_{l}^{(1)}(i \kappa r)(\mathrm{c}, \mathrm{d})$ calculated in the HCM with V1 and $m=0.3(\mathrm{a}, \mathrm{c})$ and $m=0.4(\mathrm{~b}, \mathrm{~d})$ for different cutoff hypermoments $K_{\max }$. The function $-i \kappa h_{l}^{(1)}(i \kappa r)$ is shown in the insets of panels (a) and (b).

$(2 i+1,0)$ differs only by about $0.4 \mathrm{MeV}$ from the exact solution. These calculations, shown in Fig. 1 by open squares, are lower than the HCM energies, which means that the irreps $\lambda=(2 i+1,0)$ include some important cluster excitations as well. For bound ${ }^{5} \mathrm{He}$, the difference between the full and the truncated by $\lambda=(2 i+1,0)$ HSFM calculations increases with neutron separation energy; its extrapolated value becomes $0.6 \mathrm{MeV}$ and $1.2 \mathrm{MeV}$ for $m=0.4$ and $m=0.3$, respectively. The difference between the $\lambda=(2 i+1,0)$ HSFM and the HCM calculations increases as well. This agrees with the idea that a stronger bound neutron should have more influence on the core wave function.

To investigate the long-range behavior of the valence neutron in bound ${ }^{5} \mathrm{He}$, where convergence for energy has been achieved, the radial overlap integral $I_{l j}(r)$ between the wave functions of bound ${ }^{5} \mathrm{He}$ and ${ }^{4} \mathrm{He}$ has been calculated. The definition of this overlap,

$$
\begin{aligned}
I_{l j}(r)= & \sum_{m \sigma}\left(\operatorname{lm} \frac{1}{2} \sigma \mid j m_{j}\right) \int d \hat{\boldsymbol{r}} Y_{l m}^{*}(\hat{\boldsymbol{r}}) \chi_{\frac{1}{2} \sigma \frac{1}{2} \tau}^{\dagger} \\
& \times\left\langle\Psi^{4} \mathrm{He}\left(\boldsymbol{x}_{1}, \boldsymbol{x}_{2}, \boldsymbol{x}_{3}\right) \mid \Psi_{j m_{j} \tau}^{5} \mathrm{He}\left(\boldsymbol{x}_{1}, \boldsymbol{x}_{2}, \boldsymbol{x}_{3}, \boldsymbol{r}\right)\right\rangle,
\end{aligned}
$$

includes the wave functions of ${ }^{4} \mathrm{He}$ and ${ }^{5} \mathrm{He}$ written in nonnormalized Jacobi coordinates $\boldsymbol{x}_{i}$, the last Jacobi coordinate, $\boldsymbol{x}_{4} \equiv \boldsymbol{r}$, being the distance between the valence nucleon and the center of mass of ${ }^{4} \mathrm{He}$. Such a definition is consistent with the one used in different reaction theories and in microscopic cluster models. The derivation of the final expression for this overlap is given in Sec. D of the Appendix. Because the spinorbital interaction in these calculations is not present, the total angular momentum $j$ is omitted below.

The calculated overlap $I_{l}(r)$ is shown is Figs. 2(a) and 2(b) both in linear and logarithmic scales as a function of the cutoff hypermoment $K_{\max }$, and its rms radius is shown in Fig. 3. At large $r$, the $I_{l}(r)$ should behave as

$$
\sqrt{5} I_{l}(r) \rightarrow-i C_{l} \kappa h_{1}^{(1)}(i \kappa r), \quad r \rightarrow \infty
$$

where $\kappa=\sqrt{2 \mu E_{\mathrm{rel}}} / \hbar, \mu$ is the reduced mass of ${ }^{4} \mathrm{He}+n, E_{\mathrm{rel}}$ in the neutron separation energy, equal to the binding energy difference in ${ }^{5} \mathrm{He}$ and ${ }^{4} \mathrm{He}, h_{1}^{(1)}$ is the Hankel's function of the first kind, and $C_{l}$ is the asymptotic normalization coefficient (ANC). The function $-i \kappa h_{1}^{(1)}(i \kappa r)$ is shown in the insets of Fig. 2 on a logarithmic scale and one can see that with increasing $K_{\max }$ the bevavior of the overlap $I_{l}(r)$ approaches 


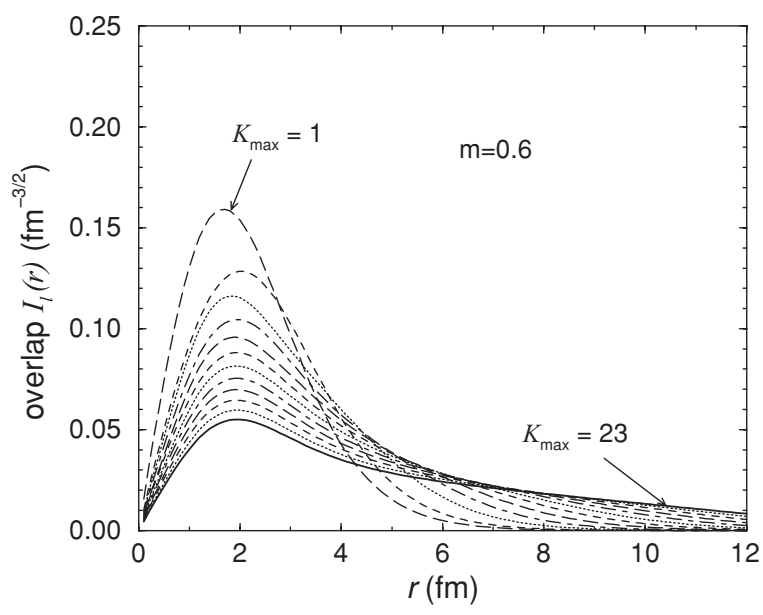

FIG. 3. The HCM overlap integral $I_{l}(r)$ for unbound ${ }^{5} \mathrm{He}$ calculated with $m=0.6$ for different cutoff hypermoments $K_{\max }$.

the trend given by Eq. (44) for both values of $m$. The ratio $5^{1 / 2} i I_{l}(r) / \kappa h_{1}^{(1)}(i \kappa r)$ is shown in Figs. 2(c) and 2(d) for $r \leqslant$ $12 \mathrm{fm}$ as a function of $K_{\max }$. For $m=0.3$, this ratio is converged at $5 \leqslant r \leqslant 7 \mathrm{fm}$, thus making unambiguous determination of $C_{l}$ possible. The $C_{l}$ value obtained at these $r$ is 0.72 $\mathrm{fm}^{-1 / 2}$. For $m=0.4,-i \kappa h_{1}^{(1)}(i \kappa r)$ has almost converged for $r \leqslant 5 \mathrm{fm}$ and $C_{l}$ determined at $r=5 \mathrm{fm}$ is $0.14 \mathrm{fm}^{-1 / 2}$. As for the $m=0.6$ case, no convergence for the overlap integral can be achieved, which is illustrated in Fig. 3, because boundary conditions are not defined in the present model.

The spectroscopic factor for the overlap $I_{l}(r)$, defined as

$$
S=5 \int_{0}^{\infty} d r r^{2} I_{l}^{2}(r)
$$

is shown in Fig. 4. For $m=0.3$, it is almost independent of $K_{\max }$ and equal to 1.2 , which is slightly lower than the value of

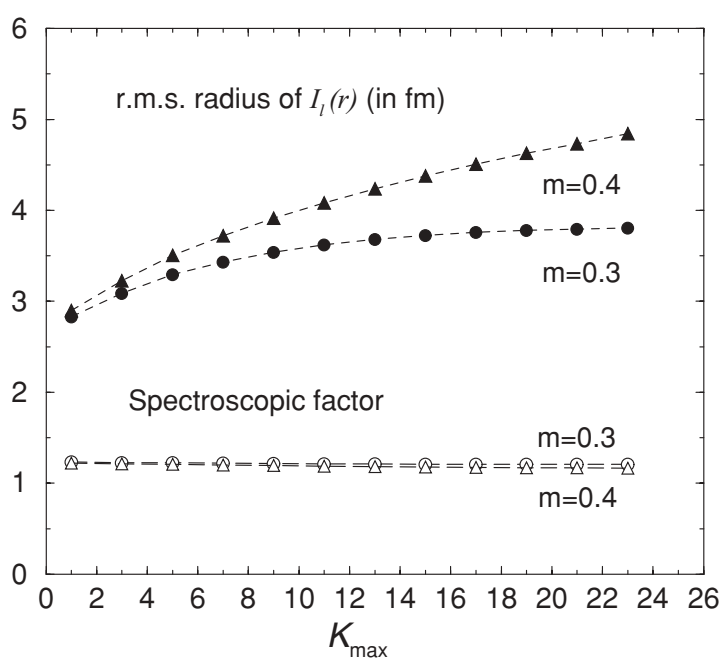

FIG. 4. The rms radius and the spectroscopic factor of the overlap integral $I_{l}(r)$ for ${ }^{5} \mathrm{He}$ calculated in the HCM for different cutoff hypermoments $K_{\max }$.

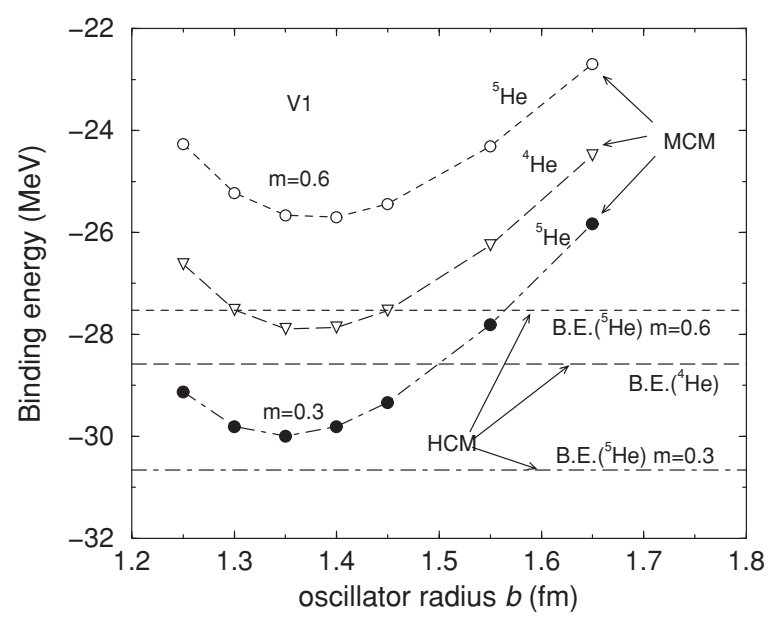

FIG. 5. Binding energy of ${ }^{5} \mathrm{He}$ calculated in the MCM for various oscillator radii $b$ in comparison with $\mathrm{HCM}$ values.

1.25 obtained in the translation-invariant oscillator $0 \hbar \omega$ shell model. For $m=0.4, S$ slowly decreases from 1.25 to 1.06 for $1 \leqslant K_{\max } \leqslant 23$. Further decrease to $S=1$ is expected with increase of $K_{\max }$.

The expansion of the ${ }^{5} \mathrm{He}$ wave function onto the $\mathrm{HCH}$ basis, given by Eq. (40), should provide very similar results to the traditional microscopic cluster model

$$
\Psi^{5} \mathrm{He}=\mathcal{A}\left(\Psi^{4} \mathrm{He} \otimes g_{l}(r) Y_{l m}(\hat{\boldsymbol{r}}) \chi_{\frac{1}{2} \sigma \frac{1}{2} \tau}\right),
$$

with the closed $0 s$-shell oscillator wave function $\Psi^{4} \mathrm{He}$. In the present article, the total binding energy of ${ }^{5} \mathrm{He}$ has been calculated in such an MCM as well for different oscillator radii $b$ and the results are compared to those calculated in the HCM in Fig. 5 for two values of the Majorana parameter, $m=0.6$ and $m=0.3$. For $m=0.3$, the MCM binding energy of ${ }^{5} \mathrm{He}$ taken at its minumum, $E=-29.99 \mathrm{MeV}$, is about $0.7 \mathrm{MeV}$ higher than the HCM result $E=-30.66 \mathrm{MeV}$. This is because the ${ }^{4} \mathrm{He}$ energy obtained in the lowest order approximation of the HSFM, $E=-28.58 \mathrm{MeV}$, is by the same amount lower than the expectation value $E=-27.89 \mathrm{MeV}$ for the ${ }^{4} \mathrm{He}$ energy in the oscillator $0 s$ shell model basis. The neutron separation energy in both cases is practically the same, $2.08 \mathrm{MeV}$ in HCM and 2.11 MeV in MCM. The HCM and MCM overlap integrals are indistinguishable when plotted in a graph. The spectroscopic factor is 1.2 for both cases and the ANC is $0.73 \mathrm{fm}^{-1 / 2}$ for MCM as compared to the HCM value of $0.72 \mathrm{fm}^{-1 / 2}$. The HCM and MCM functions $g_{l}(r)$ are also very similar. The $g_{l}(r)$ has also the asymptotic behavior given by Eq. (44) with the same ANC; however, $g_{l}(r)$ reaches the asymptotic form at significantly larger radii, thus being less convenient to use for the ANC determination. For $m=0.6$, the results of the MCM and HCM cannot be compared directly because boundary conditions for unbound states have been not formulated in the HCM. Therefore, Fig. 5 compares the MCM resonance energy with the HCM energy obtained with $K_{\max }=23$. The HCM energy is lower. As for $m=0.4$, the MCM separation energy, $E_{\text {rel }}=180 \mathrm{KeV}$, is only $40 \mathrm{keV}$ lower than the extrapolated $\mathrm{HCM}$ value of $140 \mathrm{KeV}$. As the result, the 
MCM value for ANC $C_{l}=0.16 \mathrm{fm}^{-1 / 2}$ is larger than the HCM estimate of $0.140 \mathrm{fm}^{-1 / 2}$. However, if the Majorana parameter $m$ is changed to 0.403 , then the MCM relative energy goes down to $140 \mathrm{KeV}$ and the corresponding ANC decreases to $0.142 \mathrm{fm}^{-1 / 2}$, which agrees well with the HCM calculations. The MCM spectroscopic factor for $m=0.4$ is equal to one, which is expected to be achieved by fully converged HCM calculations.

\section{SUMMARY AND CONCLUSIONS}

This work has demonstrated that the HH basis can be reorganized in such a way as to represent the cluster structure of weakly bound nuclei and to be suitable to treat the long-range behavior of valence nucleons. This can be achieved by antisymmetrizing the product of completely antisymmetric $\mathrm{HH}$ for the core and the relative hyperangular function of the last nucleon. If only a few HHs for the core are left in the expansion onto such a basis while all the quantum numbers related to the valence nucleon are retained, then such a $\mathrm{HH}$ expansion strongly resembles traditional microscopic cluster models. However, unlike in these models, the HCM wave function is not a product of the core and the relative wave functions and, as a result, the motion of the valence nucleons is not decoupled from the motion of the core nucleons. In the HCM, the core wave function depends on the position of the last nucleon. The factorization into core and relative wave functions can be achieved in the HCM by projection onto eigenfunctions of the $A-1$-body Hamiltonian. This makes the HCM to be equivalent to a multichannel microscopic cluster model with more complicated core structure and with all possible monopole core excitations built on HHs retained in the $\mathrm{HCH}$ expansion. On the other hand, the HCM is still just a version of the traditional HSFM because its hyperradial part is found by solving the same coupled set of differential equations on collective variable $\rho$ as in the HSFM.

The applicability of the HCM to treat long-range behavior has been studied using ${ }^{5} \mathrm{He}$ as an example. With only one $\mathrm{HH}$ for the ${ }^{4} \mathrm{He}$ core in the $\mathrm{HCH}$ expansion, such a model is very close to the microscopic cluster model in which the core wave function is described by the lowest approximation of the HSFM because all the monopole hyperradial excitations lie too high in energy. The calculations, performed with a simple soft-core V1 potential, have confirmed that it is possible to get a converged solution for binding energy, rms radius, overlap integral, and ANC if the strength of the Majorana force is modified to bind ${ }^{5} \mathrm{He}$. The number of basis states needed to achieve convergence depends on neutron separation energy, $12 \mathrm{HHs}$ are sufficient for $E_{\mathrm{rel}} \approx 2 \mathrm{MeV}$ but another few are needed for much smaller energies, $E_{\text {rel }} \approx 140 \mathrm{keV}$. The HCM relative energies, overlap integrals, and spectroscopic factors are very close to those calculated in the traditional MCM with the oscillator radius that gives the minimum energy in ${ }^{4} \mathrm{He}$, as expected. For unbound ${ }^{5} \mathrm{He}$, the convergence of the $\mathrm{HCH}$ expansion cannot be achieved because boundary conditions are not formulated for the HCM wave function. A scattering problem should be solved in this case rather than a bound state problem.
The long-range behavior of the last (bound) nucleon, governed by the Hankel function, is well reproduced for $m=$ 0.3 corresponding to a neutron separation energy of $2 \mathrm{MeV}$. Twelve $\mathrm{HCH}$ basis states are enough to derive the ANC with sufficient accuracy, although a few more functions are needed if better precision is required for $I_{l}(r)$ at large distances. It is important to mention that the asymptotic tail in the cluster channel is obtained correctly, although it was not assumed anyhow in the differential equations for the five-body problem. Thus, for the first time the clusterization aspect of the system dynamics is obtained by basis convergence only.

In the present test study, the $\mathrm{HCH}$ expansion has been truncated to include only the simplest ${ }^{4} \mathrm{He}$ core structure. As the result, the HCM energy converges as expected to a higher energy with respect to exact solution with the same potential. The missing energy depends on how strongly the valence neutron is bound, being about 3 and $2 \mathrm{MeV}$ for $E_{\text {rel }}=2 \mathrm{MeV}$ and $E_{\text {rel }}=140 \mathrm{KeV}$, respectively. This missing energy makes up about $10 \%$ of the total five-body energy of $\sim 30 \mathrm{MeV}$. To gain the missing energy, a more complicated structure of the core should be included, for example, particle-hole excitations. This can be done straightforwardly using the formulas from Sec. VI and from Sec. C of the Appendix. Currently, the only missing input quantities for such calculations are the Fractional Parentage Coefficients (FPCs). However, they can be derived either by using the recursive procedure of Ref. [19] or by overlapping the HHs obtained in the shell model approach developed in Ref. [14].

The present calculations have been performed using a very soft effective $N N$ potential and it is clear that realistic calculations would require much larger spaces. For ${ }^{5} \mathrm{He}$, their size can be estimated as at least ten times larger than for the ${ }^{4} \mathrm{He}$ core assuming that about ten relative hyperangular functions are required on the top of each HHs needed to get a converged wave function for ${ }^{4} \mathrm{He}$. Whether this is feasible from the point of view of modern computational power depends on how much the size of the ${ }^{4} \mathrm{He}$ model space can be reduced. However, instead of solving the system of differential equations given by Eq. (13), the $\mathrm{HCH}$ basis can be used in the hyperspherical interpolation approach of Refs. [9] and [11], where asymptotic conditions are incorporated into the system of radial equations, making the size of the basis smaller. Then the basis size for ${ }^{5} \mathrm{He}$ will be the same as that for ${ }^{4} \mathrm{He}$. A further decrease of model space can be achieved if correlated HCHs are introduced.

Finally, the ideas presented in this article can be extended to nuclei with two loosely bound valence nucleons, and in particular to Borromean nuclei. This would include more complicated matrix elements and fractional parentage expansions but would make it possible to achieve a proper three-body description of such systems at large distances within a many-body formalism.

\section{ACKNOWLEDGMENTS}

I am grateful to I. J. Thompson for providing me with his computer code STURMXX and to P. Descouvemont for his help with the MCM calculations. The EPSRC, Grant GR/T28577/01, is also acknowledged for its partial support. 


\section{APPENDIX}

\section{A. Expansion of Jacobi polynomials into oscillator wave} functions

The Jacobi polynomial $P_{n}^{\alpha, \beta}(\cos 2 \theta)$ can be represented as follows:

$$
\begin{aligned}
P_{n}^{\alpha, \beta}\left(1-2 \xi^{2} / \rho^{2}\right)= & \sum_{\nu=0}^{n}(-)^{\nu}\left(\begin{array}{c}
n+\alpha \\
n-v
\end{array}\right) \\
& \times\left(\begin{array}{c}
n+\alpha+\beta+v \\
n+\alpha+\beta
\end{array}\right) \frac{\xi^{2 v}}{\rho^{2 v}} .
\end{aligned}
$$

On the other hand,

$$
\begin{aligned}
\xi^{l+2 v} e^{-\xi^{2} / 2 b^{2}}= & b^{2 v+l+3 / 2} v ! \sum_{\kappa=0}^{v}(-1)^{\kappa}\left(\begin{array}{c}
v+l+1 / 2 \\
\kappa+l+1 / 2
\end{array}\right) \\
& \times \sqrt{\frac{\Gamma\left(\kappa+l+\frac{3}{2}\right)}{2 \kappa !}} R_{\kappa l}(\xi),
\end{aligned}
$$

where

$$
R_{\kappa l}(\xi)=b^{-3 / 2} \sqrt{\frac{2 \kappa !}{\Gamma\left(\kappa+l+\frac{3}{2}\right)}}\left(\frac{\xi}{b}\right)^{l} e^{-\xi^{2} / 2 b^{2}} L_{\kappa}^{l+1 / 2}\left(\xi^{2} / b^{2}\right)
$$

is the radial oscillator wave function with an arbitrary oscillator radius $b$ and $L_{\kappa}^{l+1 / 2}$ is the generalized Laguerre polynomial. Equation (A2) can be proven by induction. Inserting Eqs. (A2) and (A3) into Eq. (A1) gives the relation between the Jacobi polynomials and the oscillator wave functions.

\section{B. Normalization of hyperspherical cluster harmonics}

To calculate the exchange term in Eq. (32) the fractional parentage expansion of the oscillator wave function of the core can be used both in bra and ket vectors. This reads

$$
\begin{aligned}
& \Psi_{0 K_{c} \gamma_{c}}^{M_{L_{c}} M_{S_{c}} M_{T_{c}}}\left(\boldsymbol{\xi}_{1}, \ldots, \boldsymbol{\xi}_{A-2}\right) \\
& =\sum_{N^{\prime \prime} \gamma^{\prime \prime} \kappa_{1} l_{1}}\left\langle A-1 N_{c} K_{c} \gamma_{c} \mid A-2 N^{\prime \prime} K^{\prime \prime} \gamma^{\prime \prime}, \kappa_{1} l_{1}\right\rangle \\
& \quad \times \sum_{M_{L}^{\prime \prime} M_{S}^{\prime \prime} M_{T}^{\prime \prime} m_{1} \sigma_{1} \tau_{1}}\left|A-2 N^{\prime \prime} K^{\prime \prime} \gamma^{\prime \prime} M_{L}^{\prime \prime} M_{S}^{\prime \prime} M_{T}^{\prime \prime}\right\rangle \\
& \quad \times\left(L^{\prime \prime} M_{L}^{\prime \prime} l_{1} m_{1} \mid L_{c} M_{L_{c}}\right)\left(S^{\prime \prime} M_{S}^{\prime \prime} \frac{1}{2} \sigma_{1} \mid S_{c} M_{S_{c}}\right) \\
& \quad \times\left(T^{\prime \prime} M_{T}^{\prime \prime} \frac{1}{2} \tau_{1} \mid T_{c} M_{T_{c}}\right) \psi_{\kappa_{1} l_{1} m_{1}}\left(\xi_{A-2}\right) \chi_{\sigma_{1} \tau_{1}}(A-1),
\end{aligned}
$$

where $\left|A-2 N^{\prime \prime} K^{\prime \prime} \gamma^{\prime \prime} M_{L}^{\prime \prime} M_{S}^{\prime \prime} M_{T}^{\prime \prime}\right\rangle$ is a translation-invariant shell model wave function for $A-2$ nucleons with total number of oscillator quanta equal to $N^{\prime \prime}$, well-defined hypermoment $K^{\prime \prime}$, and other quantum numbers denoted by $\gamma^{\prime \prime}$. The fractional parentage coefficients (FPCs) $\left\langle A-1 N_{c} K_{c} \gamma_{c}\right| A-$ $\left.2 N^{\prime \prime} K^{\prime \prime} \gamma^{\prime \prime}, \kappa_{1} l_{1}\right\rangle$ for this expansion have been introduced in Ref. [20]. In Eq. (A4) $N_{c}$ is the total number of oscillator quanta and $N_{c}=K_{c}=K^{\prime \prime}+2 \kappa_{1}+l_{1}$. These FPCs can be calculated using the ideas of Ref. [19]. Alternatively, they can be derived by explicit overlapping shell model wave functions with well-defined hypermoments, constructed in Ref. [14], for $A-1$ and $A-2$ nucleons. It could even be more practical to expand the wave function $\Psi_{0 K_{c} \gamma_{c}}^{M_{L_{C}} M_{S_{C}} M_{T_{c}}}\left(\boldsymbol{\xi}_{1}, \ldots, \boldsymbol{\xi}_{A-2}\right)$ into wave functions for $A-2$ without a well-defined value of hypermoment. Therefore, below, the index $K^{\prime \prime}$ in the wave functions for $A-2$ nucleons in all FPCs are omitted. The index $N_{c}$ is omitted as well because $N_{c}=K_{c}$.

The wave function of the two last nucleons in the bra vector of the exchange term (32) is expressed in coordinates $\boldsymbol{\xi}_{A-2}$ and $\boldsymbol{\xi}_{A-1}$ while in the ket-vertor it is expressed in $\boldsymbol{\xi}_{A-2}^{\prime}$ and $\xi_{A-1}^{\prime}$,

$$
\begin{aligned}
& \xi_{A-2}^{\prime}=\sqrt{\frac{A-2}{A-1}}\left(\frac{1}{A-2} \sum_{i=1}^{A-2} \boldsymbol{r}_{i}-\boldsymbol{r}_{A}\right), \\
& \xi_{A-1}^{\prime}=\sqrt{\frac{A-1}{A}}\left(\frac{1}{A-1}\left(\sum_{i=1}^{A-2} \boldsymbol{r}_{i}+\boldsymbol{r}_{A}\right)-\boldsymbol{r}_{A-1}\right) .
\end{aligned}
$$

Using the Talmi-Moshinsky transformation

$$
\begin{aligned}
& \left|\psi_{\kappa_{1} l_{1}}\left(\boldsymbol{\xi}_{A-2}\right) \psi_{\kappa l}\left(\boldsymbol{\xi}_{A-1}\right): L_{0} M_{0}\right\rangle \\
& =\sum_{\kappa_{1} l_{1} \kappa_{1}^{\prime} l_{1}^{\prime}}\left\langle\kappa^{\prime} l^{\prime} \kappa_{1}^{\prime} l_{1}^{\prime}: L_{0}|A(A-2)| \kappa_{1} l_{1} \kappa l: L_{0}\right\rangle \\
& \quad \times\left|\psi_{\kappa^{\prime} l^{\prime}}\left(\xi_{A-1}^{\prime}\right) \psi_{\kappa_{1}^{\prime} l_{1}^{\prime}}\left(\xi_{A-2}^{\prime}\right): L_{0} M_{0}\right\rangle,
\end{aligned}
$$

where $\left\langle\kappa^{\prime} l^{\prime} \kappa_{1}^{\prime} l_{1}^{\prime}: L_{0}|A(A-2)| \kappa_{1} l_{1} \kappa l: L_{0}\right\rangle$ is the TalmiMoshinsky coefficient (the ordering and meaning of symbols in this coefficient are the same as in Ref. [21]) and $2 \kappa_{1}+l_{1}+$ $2 \kappa+l=2 \kappa_{1}^{\prime}+l_{1}^{\prime}+2 \kappa^{\prime}+l^{\prime}$, we get for the overlap $I_{\alpha \alpha^{\prime}}^{S M}$ the following expression:

$$
\begin{aligned}
& I_{\alpha \alpha^{\prime}}^{S M}=\left\langle\mathcal{A} \Phi_{\alpha^{\prime}}^{(b)} \mid \mathcal{A} \Phi_{\alpha}^{(b)}\right\rangle=\delta_{\alpha \alpha^{\prime}}-\delta_{L L^{\prime}} \delta_{S S^{\prime}} \delta_{T T^{\prime}}(A-1) \\
& \times \sum_{N^{\prime \prime} \gamma^{\prime \prime}} U\left(S^{\prime \prime} \frac{1}{2} \frac{1}{2} S ; S_{c} S_{c}^{\prime}\right) U\left(T^{\prime \prime} \frac{1}{2} \frac{1}{2} T ; T_{c} T_{c}^{\prime}\right) \\
& \times(-)^{S+S^{\prime \prime}+S_{c}+S_{c}^{\prime}+T+T^{\prime \prime}+T_{c}+T_{c}^{\prime}} \\
& \times \sum_{\kappa_{1} l_{1} \kappa_{1}^{\prime} l_{1}^{\prime}}\left\langle A-1 K_{c} \gamma_{c} \mid A-2 N^{\prime \prime} \gamma^{\prime \prime}, \kappa_{1} l_{1}\right\rangle \\
& \times\left\langle A-1 K_{c}^{\prime} \gamma_{c}^{\prime} \mid A-2 N^{\prime \prime} \gamma^{\prime \prime}, \kappa_{1}^{\prime} l_{1}^{\prime}\right\rangle \\
& \times \sum_{L_{0}}(-)^{l^{\prime}+l_{1}^{\prime}-L_{0}} U\left(L^{\prime \prime} l_{1} L l ; L_{c} L_{0}\right) U\left(L^{\prime \prime} l_{1}^{\prime} L l^{\prime} ; L_{c}^{\prime} L_{0}\right) \\
& \times\left\langle\kappa^{\prime} l^{\prime} \kappa_{1}^{\prime} l_{1}^{\prime}: L_{0}|A(A-2)| \kappa_{1} l_{1} \kappa l: L_{0}\right\rangle,
\end{aligned}
$$

where $U\left(j_{1} j_{2} J j_{3} ; j_{12} j_{23}\right)=\hat{j}_{12} \hat{j}_{23} W\left(j_{1} j_{2} J j_{3} ; j_{12} j_{23}\right), \hat{j}=$ $\sqrt{2 j+1}$, and $W$ is the Racah coefficient.

\section{Matrix elements of central two-body $N \boldsymbol{N}$ interactions}

The matrix elements $\left\langle\mathcal{A} \psi_{\alpha^{\prime}}|V| \mathcal{A} \psi_{\alpha}\right\rangle$ between the antisymmetrized oscillator cluster shell model wave functions contain direct and exchange terms,

$$
\left\langle\mathcal{A} \psi_{\alpha^{\prime}}|V| \mathcal{A} \psi_{\alpha}\right\rangle=V_{\mathrm{dir}}-(A-1) V_{\mathrm{ex}} .
$$

The direct term

$$
\begin{aligned}
V_{\mathrm{dir}}= & \left\langle\phi_{\alpha_{1}^{\prime}}(1, \ldots, A-1) \varphi_{\alpha_{2}^{\prime}}(A)|V|\right. \\
& \left.\times \phi_{\alpha_{1}}(1, \ldots, A-1) \varphi_{\alpha_{2}}(A)\right\rangle
\end{aligned}
$$


can be separated into two parts,

$$
\begin{aligned}
V_{\mathrm{dir}}= & V_{\mathrm{dir}}^{(1)}+V_{\mathrm{dir}}^{(2)}=\delta_{\alpha_{2}, \alpha_{2}^{\prime}}\left|\phi_{\alpha_{1}^{\prime}}(1, \ldots, A-1)\right| \\
& \times \sum_{i<j}^{A-1} V_{i j}\left|\phi_{\alpha_{1}}(1, \ldots, A-1)\right\rangle \\
& +\left\langle\phi_{\alpha_{1}^{\prime}}(1, \ldots, A-1) \varphi_{\alpha_{2}^{\prime}}(A)\right| \\
& \times \sum_{i=1}^{A-1} V_{i A}\left|\phi_{\alpha_{1}}(1, \ldots, A-1) \varphi_{\alpha_{2}}(A)\right\rangle
\end{aligned}
$$

the first of which is the expectation value of the $N N$ potential for $A-1$ nucleons of the core in the standard translationinvariant shell model basis,

$$
\begin{aligned}
V_{\mathrm{dir}}^{(1)}= & \delta_{\kappa^{\prime} \kappa} \delta_{l l^{\prime}} \delta_{m m^{\prime}}\left\langle\Psi_{0 K_{0}^{\prime} \gamma_{0}^{\prime}}^{M_{L_{0}}^{\prime} M_{S_{0}}^{\prime} M_{T_{0}}^{\prime}}\left(\boldsymbol{\xi}_{1}, \ldots, \boldsymbol{\xi}_{A-2}\right)\right| \\
& \times \sum_{i<j}^{A-1} V_{i j}\left|\Psi_{0 K_{0} \gamma_{0}}^{M_{L_{0}} M_{S_{0}} M_{T_{0}}}\left(\boldsymbol{\xi}_{1}, \ldots, \boldsymbol{\xi}_{A-2}\right)\right\rangle,
\end{aligned}
$$

and the second term $V_{\mathrm{dir}}^{(2)}$ being the folding potential between the last nucleon and the core. The exchange potential

$$
\begin{aligned}
V_{\mathrm{ex}}= & \left\langle\phi_{\alpha_{1}^{\prime}}(1, \ldots, A) \varphi_{\alpha_{2}^{\prime}}(A-1)|V|\right. \\
& \left.\times \phi_{\alpha_{1}}(1, \ldots, A-2, A-1), \varphi_{\alpha_{2}}(A)\right\rangle
\end{aligned}
$$

can be represented by four terms,

$$
V_{\mathrm{ex}}=V_{\mathrm{ex}}^{(1)}+V_{\mathrm{ex}}^{(2)}+V_{\mathrm{ex}}^{(3)}+V_{\mathrm{ex}}^{(4)},
$$

according to the following separation of the two-body interaction potential:

$$
\sum_{i<j}^{A} V_{i j}=V_{A-1, A}+\sum_{i=1}^{A-2} V_{i A}+\sum_{i=1}^{A-2} V_{i A-1}+\sum_{i<j}^{A-2} V_{i j}
$$

The terms $V_{\mathrm{dir}}^{(2)}$ and $V_{\mathrm{ex}}^{(1)}$ resemble each other. They can be easily calculated by separating the wave function of the $(A-1, A)$ pair in the cluster wave function $\Phi_{\alpha}^{(b)}$. This can be achieved by using the fractional parentage expansion (A4) of the core wave function $\Psi_{0 K_{c} \gamma_{c}}^{M_{L_{c}} M_{S_{c}} M_{T_{C}}}\left(\xi_{1}, \ldots, \xi_{A-2}\right)$ combined with the Talmi-Moshinsky transformation

$$
\begin{aligned}
& \left|\psi_{\kappa_{1} l_{1}}\left(\xi_{A-2}\right) \psi_{\kappa l}\left(\xi_{A-1}\right): L_{0} M_{0}\right\rangle \\
& =\sum_{n_{0} l_{0} N \Lambda}\left\langle\kappa_{1} l_{1} \kappa_{1} l_{1}: L_{0}\left|\frac{A-2}{A}\right| n_{0} l_{0} N \Lambda: L_{0}\right\rangle \\
& \quad \times\left|\psi_{n_{0} l_{0}}\left(\zeta_{1}\right) \psi_{N \Lambda}\left(\zeta_{2}\right): L_{0} M_{0}\right\rangle,
\end{aligned}
$$

in which

$$
\begin{aligned}
& \zeta_{1}=\frac{1}{\sqrt{2}}\left(\boldsymbol{r}_{A-1}-\boldsymbol{r}_{A}\right), \\
& \zeta_{2}=\sqrt{\frac{2(A-2)}{A}}\left(\frac{1}{A-2} \sum_{i=1}^{A-2} \boldsymbol{r}_{i}-\frac{\boldsymbol{r}_{A-1}+\boldsymbol{r}_{A}}{2}\right) .
\end{aligned}
$$

Then the wave function $\Phi_{\alpha}^{(b)}$ reads as follows

$$
\begin{aligned}
& \Phi_{K_{c} \gamma_{c} \kappa l L S T M_{L} M_{S} M_{T}}^{(b)}\left(\xi_{1}, \ldots \xi_{A-1}\right) \\
& =\sum_{N^{\prime \prime} \gamma^{\prime \prime} \kappa_{1} l_{1} M_{L}^{\prime \prime} M_{S}^{\prime \prime} M_{T}^{\prime \prime}}\left|A-2 N^{\prime \prime} \gamma^{\prime \prime} M_{L}^{\prime \prime} M_{S}^{\prime \prime} M_{T}^{\prime \prime}\right\rangle
\end{aligned}
$$

$$
\begin{aligned}
& \times\left\langle A-1 K_{c} \gamma_{c} \mid A-2 N^{\prime \prime} \gamma^{\prime \prime}, \kappa_{1} l_{1}\right\rangle \\
& \times \sum_{n_{0} l_{0} N \Lambda L_{0}}\left\langle\kappa_{1} l_{1}, \kappa l: L_{0}\left|\frac{A-2}{A}\right| n_{0} l_{0}, N \Lambda: L_{0}\right\rangle \\
& \times\left|\psi_{n_{0} l_{0}}\left(\zeta_{1}\right), \psi_{N \Lambda}\left(\zeta_{2}\right): L_{0} M_{L_{0}}\right| U\left(L^{\prime \prime} l_{1} L l ; L_{c} L_{0}\right) \\
& \times \sum_{S_{0} T_{0}} \chi_{S_{0} M_{S_{0}} T_{0} M_{T_{0}}}(A-1, A) \\
& \times U\left(S^{\prime \prime} \frac{1}{2} S \frac{1}{2} ; S_{c} S_{0}\right) U\left(T^{\prime \prime} \frac{1}{2} T \frac{1}{2} ; T_{c} T_{0}\right) \\
& \times \sum_{M_{L_{0}} M_{S_{0}} M_{T_{0}}}\left(L^{\prime \prime} M_{L}^{\prime \prime} L_{0} M_{L_{0}} \mid L M_{L}\right)\left(S^{\prime \prime} M_{S}^{\prime \prime} S_{0} M_{S_{0}} \mid S M_{S}\right) \\
& \times\left(T^{\prime \prime} M_{T}^{\prime \prime} T_{0} M_{T_{0}} \mid T M_{T}\right) .
\end{aligned}
$$

The expansion of the wave function $\Phi_{\alpha^{\prime}}^{(b)}$ in the bra vector looks exactly the same apart from an additional phase factor $(-)^{l_{0}+S_{0}+T_{0}}$ that appears due to interchange of $A-1$-th and $A$-th nucleons. As the result, we get

$$
\begin{aligned}
V_{\mathrm{dir}}^{(2)} & -(A-1) V_{\mathrm{ex}}^{(1)} \\
= & (A-1)\left\langle\Phi_{\alpha^{\prime}}^{(b)}(1, \ldots, A-1, A)\right. \\
& \left.-\Phi_{\alpha^{\prime}}^{(b)}(1, \ldots, A, A-1)\left|V_{A-1, A}\right| \Phi_{\alpha}^{(b)}(1, \ldots, A-1, A)\right\rangle \\
= & (A-1) \sum_{n_{0}^{\prime} n_{0} l_{0} s t}\left(1-(-1)^{l_{0}+s+t}\right) C_{n_{0}^{\prime} n_{0} l_{0} s t}^{\alpha^{\prime}} \\
& \times\left\langle\psi_{n_{0}^{\prime} l_{0}}\left\|V^{(s t)}\right\| \psi_{n_{0} l_{0}}\right\rangle,
\end{aligned}
$$

where

$$
\begin{aligned}
C_{n_{0}^{\prime} n_{0} l_{0} s t}^{\alpha^{\prime} \alpha}= & \sum_{N^{\prime \prime} \gamma^{\prime \prime} \kappa_{1} l_{1} \kappa_{1}^{\prime} l_{1}^{\prime}}\left\langle A-1 K_{c} \gamma_{c} \mid A-2 N^{\prime \prime} \gamma^{\prime \prime}, \kappa_{1} l_{1}\right\rangle \\
& \times\left\langle A-1 K_{c}^{\prime} \gamma_{c}^{\prime} \mid A-2 N^{\prime \prime} \gamma^{\prime \prime}, \kappa_{1}^{\prime} l_{1}^{\prime}\right\rangle U\left(S^{\prime \prime} \frac{1}{2} S \frac{1}{2} ; S_{c} s\right) \\
& \times U\left(T^{\prime \prime} \frac{1}{2} T \frac{1}{2} ; T_{c} t\right) U\left(S^{\prime \prime} \frac{1}{2} S \frac{1}{2} ; S_{c}^{\prime} s\right) \\
& \times U\left(T^{\prime \prime} \frac{1}{2} T \frac{1}{2} ; T_{c}^{\prime} t\right) \sum_{L_{0}} U\left(L^{\prime \prime} l_{1} L l ; L_{c} L_{0}\right) \\
& \times U\left(L^{\prime \prime} l_{1}^{\prime} L l^{\prime} ; L_{c}^{\prime} L_{0}\right) \\
& \times \sum_{N \Lambda}\left\langle\kappa_{1} l_{1}, \kappa l: L_{0}\left|\frac{A-2}{A}\right| n_{0} l_{0}, N \Lambda: L_{0}\right\rangle \\
& \times\left\langle\kappa_{1}^{\prime} l_{1}^{\prime}, \kappa^{\prime} l^{\prime}: L_{0}\left|\frac{A-2}{A}\right| n_{0}^{\prime} l_{0}, N \Lambda: L_{0}\right\rangle .
\end{aligned}
$$

To calculate the exchange term $V_{\mathrm{ex}}^{(2)}$,

$$
\begin{aligned}
V_{\mathrm{ex}}^{(2)}= & \left\langle\phi_{\alpha_{1}^{\prime}}(1, \ldots, A) \varphi_{\alpha_{2}^{\prime}}(A-1)\right| \\
& \times \sum_{i=1}^{A-2} V_{i A}\left|\phi_{\alpha_{1}}(1, \ldots, A-2, A-1) \varphi_{\alpha_{2}}(A)\right\rangle \\
= & (A-2)\left\langle\phi_{\alpha_{1}^{\prime}}(1, \ldots, A-2, A) \varphi_{\alpha_{2}^{\prime}}(A-1)\left|V_{A-2, A}\right|\right. \\
& \left.\times \phi_{\alpha_{1}}(1, \ldots, A-2, A-1) \varphi_{\alpha_{2}}(A)\right\rangle,
\end{aligned}
$$

it is convenient to separate the wave function of the pair $(A-$ $2, A-1)$ from the cluster function $\Phi_{\alpha}^{(b)}$ using a two-nucleon 
fractional parentage expansion for the core,

$$
\begin{aligned}
& \Psi_{0 K_{c} \gamma_{c}}^{M_{L_{c}} M_{S_{c}} M_{T_{c}}}\left(\xi_{1}, \ldots, \xi_{A-2}\right)=\sum_{N^{\prime \prime} K^{\prime \prime} \gamma^{\prime \prime} N \Lambda n_{0} l_{0} L_{0} S_{0} T_{0}} \\
& \times\left\langle A-1 K_{c} \gamma_{c} \mid A-3 N^{\prime \prime} K^{\prime \prime} \gamma^{\prime \prime} ; N \Lambda, n_{0} l_{0} S_{0} T_{0}\left(L_{0}\right): L_{c} S_{c} T_{c}\right\rangle \\
& \times \sum_{M_{L}^{\prime \prime} M_{S}^{\prime \prime} M_{T}^{\prime \prime} M_{L_{0}} M_{S_{0}} M_{T_{0}}}\left|A-3 N^{\prime \prime} K^{\prime \prime} L^{\prime \prime} M_{L}^{\prime \prime} S^{\prime \prime} M_{S}^{\prime \prime} T^{\prime \prime} M_{T}^{\prime \prime}\right\rangle \\
& \times\left|\psi_{N \Lambda}\left(\eta_{2}\right), \psi_{n_{0} l_{0}}\left(\eta_{1}\right): L_{0} M_{L_{0}}\right\rangle\left(L^{\prime \prime} M_{L}^{\prime \prime} L_{0} M_{L_{0}} \mid L_{c} M_{L_{c}}\right) \\
& \times\left(S^{\prime \prime} M_{S}^{\prime \prime} S_{0} M_{S_{0}} \mid S_{c} M_{S_{c}}\right)\left(T^{\prime \prime} M_{T}^{\prime \prime} T_{0} M_{T_{0}} \mid T_{c} M_{T_{c}}\right) \\
& \times \chi_{S_{0} M_{S_{0}} T_{0} M_{T_{0}}}(A-2, A),
\end{aligned}
$$

where $\left|A-3 N^{\prime \prime} K^{\prime \prime} L^{\prime \prime} M_{L}^{\prime \prime} S^{\prime \prime} M_{S}^{\prime \prime} T^{\prime \prime} M_{T}^{\prime \prime}\right\rangle$ is the translationinvariant shell model wave function for $A-3$ nucleons with $N^{\prime \prime}$ quanta, hypermoment $K^{\prime \prime}$, and other quantum numbers denoted by $\gamma^{\prime \prime}$ and $\left\langle A-1 K_{c} \gamma_{c}\right| A-$ $\left.3 N^{\prime \prime} K^{\prime \prime} \gamma^{\prime \prime} ; N \Lambda, n_{0} l_{0} S_{0} T_{0}\left(L_{0}\right): L_{c} S_{c} T_{c}\right\rangle$ is the two-nucleon FPC. As explained in the previous section, $K^{\prime \prime}$ is omitted below. In Eq. (A21), the coordinates $\eta_{1}$ and $\eta_{2}$ are the following,

$$
\begin{aligned}
& \boldsymbol{\eta}_{1}=\frac{1}{\sqrt{2}}\left(\boldsymbol{r}_{A-2}-\boldsymbol{r}_{A-1}\right), \\
& \boldsymbol{\eta}_{2}=\sqrt{\frac{2(A-3)}{A-1}}\left(\frac{1}{A-3} \sum_{i=1}^{A-3} \boldsymbol{r}_{i}-\frac{\boldsymbol{r}_{A-2}+\boldsymbol{r}_{A-1}}{2}\right) .
\end{aligned}
$$

Exchange of nucleons $A-1$ and $A$ leads to new coordinates, $\boldsymbol{\eta}_{1}^{\prime}$ and $\boldsymbol{\eta}_{2}^{\prime}$, that are obtained from $\boldsymbol{\eta}_{1}$ and $\boldsymbol{\eta}_{2}$ by replacing $\boldsymbol{r}_{A-1}$ by $\boldsymbol{r}_{A}$. Applying the expansion (A21) and using the transformation

$$
\begin{aligned}
& \sum_{M_{L_{0}} M_{L_{c}} m}\left(L^{\prime \prime} M_{L}^{\prime \prime} L_{0} M_{L_{0}} \mid L_{c} M_{L_{c}}\right)\left(L_{c} M_{L_{c}} \operatorname{lm} \mid L M\right) \\
& \left.\quad \times \mid \psi_{N \Lambda}\left(\boldsymbol{\eta}_{2}\right), \psi_{n_{0} l_{0}}\left(\boldsymbol{\eta}_{1}\right): L_{0} M_{L_{0}}\right) \psi_{\kappa l m}\left(\xi_{A-1}\right) \\
& =\sum_{L_{0}^{\prime \prime} M_{L_{0}}^{\prime \prime} L_{c}^{\prime \prime} M_{L_{c}}^{\prime \prime} \kappa^{\prime \prime} l^{\prime \prime} m^{\prime \prime} N^{\prime} \Lambda^{\prime} n_{0}^{\prime} l_{0}^{\prime}}\left(L^{\prime \prime} M_{L}^{\prime \prime} L_{0}^{\prime \prime} M_{L_{0}}^{\prime \prime} \mid L_{c}^{\prime \prime} M_{L_{c}}^{\prime \prime}\right) \\
& \quad \times\left(L_{c}^{\prime \prime} M_{L_{c}}^{\prime \prime} l^{\prime \prime} m^{\prime \prime} \mid L M\right)\left|\psi_{N^{\prime} \Lambda^{\prime}}\left(\eta_{2}^{\prime}\right), \psi_{n_{0}^{\prime} l_{0}^{\prime}}\left(\boldsymbol{\eta}_{1}^{\prime}\right): L_{0}^{\prime \prime} M_{L_{0}}^{\prime \prime}\right\rangle \\
& \quad \times \psi_{\kappa^{\prime \prime} l^{\prime \prime} m^{\prime}}\left(\boldsymbol{\xi}_{A-1}^{\prime}\right) T_{L^{\prime \prime}, L_{0} L_{c} \kappa l, L_{0}^{\prime \prime} L_{c}^{\prime \prime} \kappa^{\prime \prime} l^{\prime \prime}, L}^{N \Lambda n_{0} l_{0}, N^{\prime} \Lambda^{\prime} n^{\prime} l^{\prime}},
\end{aligned}
$$

the wave function $\Phi_{K_{c} \gamma_{c} \kappa l L S T M_{L} M_{S} M_{T}}^{(b)}(1, \ldots, A-2, A-1, A)$ can be rewritten as

$$
\begin{aligned}
& \Phi_{K_{c} \gamma_{c} \kappa l L S T M_{L} M_{S} M_{T}}^{(b)}(1, \ldots, A-2, A-1, A) \\
& =\sum^{N^{\prime \prime} \gamma^{\prime \prime} N \Lambda N^{\prime} \Lambda^{\prime} n_{0} l_{0} n_{0}^{\prime} l_{0}^{\prime} n^{\prime \prime} l^{\prime \prime}}\left|A-3 N^{\prime \prime} \gamma^{\prime \prime} M_{L}^{\prime \prime} M_{S}^{\prime \prime} M_{T}^{\prime \prime}\right\rangle \\
& \\
& \quad L_{0} L_{0}^{\prime} L_{c}^{\prime \prime} S_{0} S_{0}^{\prime} S_{c}^{\prime \prime} T_{0} T_{0}^{\prime} T_{c}^{\prime \prime}\{\mu\} \\
& \quad \times\left\langle A-1 K_{c} \gamma_{c} \mid A-3 N^{\prime \prime} \gamma^{\prime \prime} ; N \Lambda, n_{0} l_{0} S_{0} T_{0}\left(L_{0}\right): L_{c} S_{c} T_{c}\right\rangle \\
& \quad \times\left|\psi_{N^{\prime} \Lambda^{\prime}}\left(\eta_{2}^{\prime}\right) \psi_{n_{0}^{\prime} l_{0}^{\prime}}\left(\eta_{1}^{\prime}\right): L_{0}^{\prime} M_{L_{0}}^{\prime}\right\rangle \varphi_{\kappa^{\prime \prime} l^{\prime \prime} m^{\prime \prime}}\left(\xi_{A-1}^{\prime}\right) \\
& \quad \times T_{L^{\prime \prime}, L_{0} L_{c} \kappa l, L_{0}^{\prime \prime} L_{c}^{\prime \prime} \kappa^{\prime \prime} l^{\prime \prime}, L}^{N \Lambda l_{0} l_{0}, N^{\prime} \Lambda^{\prime} n^{\prime}{ }^{\prime}} \\
& \quad \times\left(L^{\prime \prime} M_{L}^{\prime \prime} L_{0}^{\prime} M_{L_{0}}^{\prime} \mid L_{c}^{\prime \prime} M_{L_{c}}^{\prime \prime}\right)\left(L_{c}^{\prime \prime} M_{L_{c}}^{\prime \prime} l^{\prime \prime} m^{\prime \prime} \mid L M_{L}\right) \\
& \quad \times \chi_{S_{0}^{\prime} M_{S_{0}}^{\prime} T_{0}^{\prime} M_{T_{0}}^{\prime}}(A-2, A) \chi_{\sigma^{\prime} \tau^{\prime}}(A-1)
\end{aligned}
$$

$$
\begin{aligned}
& \times \hat{S}_{c} \hat{S}_{c}^{\prime \prime} \hat{S}_{0} \hat{S}_{0}^{\prime} \hat{T}_{c} \hat{T}_{c}^{\prime \prime} \hat{T}_{0} \hat{T}_{0}^{\prime}\left\{\begin{array}{ccc}
S & \frac{1}{2} & S_{c}^{\prime \prime} \\
\frac{1}{2} & \frac{1}{2} & S_{0}^{\prime} \\
S_{c} & S_{0} & S^{\prime \prime}
\end{array}\right\}\left\{\begin{array}{ccc}
T & \frac{1}{2} & T_{c}^{\prime \prime} \\
\frac{1}{2} & \frac{1}{2} & T_{0}^{\prime} \\
T_{c} & T_{0} & T^{\prime \prime}
\end{array}\right\} \\
& \times\left(S^{\prime \prime} M_{S}^{\prime \prime} S_{0}^{\prime} M_{S_{0}}^{\prime} \mid S_{c}^{\prime \prime} M_{S_{c}}^{\prime \prime}\right)\left(S_{c}^{\prime \prime} M_{S_{c}}^{\prime \prime} \frac{1}{2} \sigma^{\prime} \mid S M_{S}\right) \\
& \times\left(T^{\prime \prime} M_{T}^{\prime \prime} T_{0}^{\prime} M_{T_{0}}^{\prime} \mid T_{c}^{\prime \prime} M_{T_{c}}^{\prime \prime}\right)\left(T_{c}^{\prime \prime} M_{T_{c}}^{\prime \prime} \frac{1}{2} \sigma^{\prime} \mid T M_{T}\right),
\end{aligned}
$$

where $\{\mu\}=\left\{M_{L}^{\prime \prime} M_{S}^{\prime \prime} M_{T}^{\prime \prime} \tilde{M}_{L} \tilde{M}_{S}^{\prime} \tilde{M}_{T}^{\prime} \tilde{M}_{S_{0}} \tilde{M}_{T_{0}} M_{L_{2}} m_{1} \sigma \tau\right\}$ and

$$
\begin{aligned}
T_{L^{\prime \prime}, L_{0} L_{c} \kappa l, L_{0}^{\prime \prime} L_{c}^{\prime \prime} \kappa^{\prime \prime} l^{\prime \prime}, L}^{N} n_{0} l_{0}, N^{\prime} \Lambda^{\prime} l^{\prime} l^{\prime} & \sum_{\tilde{N} \tilde{\Lambda} \tilde{n} n_{1} l_{1} \mathcal{L} \mathcal{L}^{\prime}}\left\langle\tilde{N} \tilde{\Lambda} \tilde{n} \tilde{l}: \mathcal{L}\left|\frac{A(A-3)}{2}\right| N \Lambda \kappa l: \mathcal{L}\right\rangle \\
& \times\left\langle n_{1} l_{1} \kappa^{\prime \prime} l^{\prime \prime}: \mathcal{L}^{\prime}\left|\frac{A}{A-2}\right| \tilde{N} \tilde{\Lambda} n_{0} l_{0}: \mathcal{L}^{\prime}\right\rangle \\
& \times\left\langle n_{0}^{\prime} l_{0}^{\prime} N^{\prime} \Lambda^{\prime}: L_{0}^{\prime \prime}\left|\frac{A-3}{A-1}\right| \tilde{l} \tilde{l} n_{1} l_{1}: L_{0}^{\prime \prime}\right\rangle \\
& \times \sum_{\lambda_{c} \lambda_{c}^{\prime}}\left(\hat{\lambda}_{c}^{\prime} \hat{\mathcal{L}}^{\prime} \hat{\lambda}_{c} \hat{\mathcal{L}}\right)^{2} \hat{L}_{c}^{\prime \prime} \hat{L}_{0}^{\prime \prime} \hat{L}_{c} \hat{L}_{0} \\
& \times\left\{\begin{array}{lll}
L & l^{\prime \prime} & L_{c}^{\prime \prime} \\
\tilde{l} & l_{1} & L_{0}^{\prime \prime} \\
\lambda_{c}^{\prime} & \mathcal{L}^{\prime} & L^{\prime \prime}
\end{array}\right\}\left\{\begin{array}{lll}
L & l_{0} & \lambda_{c} \\
\tilde{l} & \tilde{\Lambda} & \mathcal{L} \\
\lambda_{c}^{\prime} & \mathcal{L}^{\prime} & L^{\prime \prime}
\end{array}\right\}\left\{\begin{array}{lll}
L & l_{0} & \lambda_{c} \\
l & \Lambda & \mathcal{L} \\
L_{c} & L_{0} & L^{\prime \prime}
\end{array}\right\} .
\end{aligned}
$$

Then in the wave function $\Phi_{K_{c}^{\prime} \gamma_{c}^{\prime} \kappa^{\prime} l^{\prime} L S T M_{L} M_{S} M_{T}}^{(b)}(1, \ldots, A-$ 2, $A, A-1)$, in which the nucleons $A-1$ and $A$ are interchanged, it is suffucient to extract the pair $(A-2, A)$. This gives

$$
\begin{aligned}
& \Phi_{K_{c}^{\prime} \gamma_{c}^{\prime} \kappa^{\prime} l^{\prime} L S T M_{L} M_{S} M_{T}}^{(b)}(1, \ldots, A-2, A, A-1) \\
& =\sum_{N^{\prime \prime} \gamma^{\prime \prime} \tilde{N}^{\prime} \tilde{\Lambda}^{\prime} n_{0}^{\prime \prime} l_{0}^{\prime \prime} L_{0}^{\prime \prime} S_{0}^{\prime \prime} T_{0}^{\prime \prime}\left\{\mu^{\prime}\right\}}\left|A-3 N^{\prime \prime} \gamma^{\prime \prime} M_{L}^{\prime \prime} M_{S}^{\prime \prime} M_{T}^{\prime \prime}\right\rangle \\
& \quad \times\left\langle A-1 K_{c}^{\prime} \gamma_{c}^{\prime} \mid A-3 N^{\prime \prime} \gamma^{\prime \prime} ; \tilde{N}^{\prime} \tilde{\Lambda}^{\prime}, n_{0}^{\prime \prime} l_{0}^{\prime \prime} S_{0}^{\prime \prime} T_{0}^{\prime \prime}\left(L_{0}^{\prime \prime}\right): L_{c}^{\prime} S_{c}^{\prime} T_{c}^{\prime}\right\rangle \\
& \quad \times\left|\psi_{\tilde{N}^{\prime}} \tilde{\Lambda}^{\prime}\left(\eta_{2}^{\prime}\right) \psi_{n_{0}^{\prime \prime} l_{0}^{\prime \prime}}\left(\eta_{1}^{\prime}\right): L_{0}^{\prime \prime} M_{L_{0}}^{\prime \prime}\right\rangle \varphi_{\kappa^{\prime} l^{\prime} m^{\prime}}\left(\xi_{A-1}^{\prime}\right) \\
& \quad \times \chi_{S_{0}^{\prime \prime} M_{S_{0}}^{\prime \prime} T_{0}^{\prime \prime} M_{T_{0}}^{\prime \prime}}(A-2, A) \chi_{\sigma \tau}(A-1) \\
& \quad \times\left(L^{\prime \prime} M_{L}^{\prime \prime} L_{0}^{\prime \prime} M_{L_{0}}^{\prime \prime} \mid L_{c}^{\prime} M_{L_{c}}^{\prime}\right)\left(L_{c}^{\prime} M_{L_{c}}^{\prime} l^{\prime \prime} m^{\prime \prime} \mid L M_{L}\right) \\
& \quad \times\left(S^{\prime \prime} M_{S}^{\prime \prime} S_{0}^{\prime \prime} M_{S_{0}}^{\prime \prime} \mid S_{c}^{\prime} M_{S_{c}}^{\prime}\right)\left(S_{c}^{\prime} M_{S_{c}}^{\prime} \frac{1}{2} \sigma \mid S M_{S}\right) \\
& \quad \times\left(T^{\prime \prime} M_{T}^{\prime \prime} T_{0}^{\prime \prime} M_{T_{0}}^{\prime \prime} \mid T_{c}^{\prime} M_{T_{c}}^{\prime}\right)\left(T_{c}^{\prime} M_{T_{c}}^{\prime} \frac{1}{2} \tau \mid T M_{T}\right), \quad \text { (A26) }
\end{aligned}
$$

where $\quad\left\{\mu^{\prime}\right\}=\left\{M_{L}^{\prime \prime} M_{S}^{\prime \prime} M_{T}^{\prime \prime} M_{L_{0}}^{\prime \prime} M_{S_{0}}^{\prime \prime} M_{T_{0}}^{\prime \prime} M_{L_{c}}^{\prime} M_{S_{c}}^{\prime} M_{T_{c}}^{\prime} m^{\prime \prime} \sigma \tau\right\}$. Using Eqs. (A24) and (A26) in Eq. (A20) gives the exchange term $V_{\mathrm{ex}}^{(2)}$. The exchange term $V_{\mathrm{ex}}^{(3)}$,

$$
\begin{aligned}
V_{\mathrm{ex}}^{(3)}= & \left\langle\phi_{\alpha_{1}^{\prime}}(1, \ldots, A-2, A) \varphi_{\alpha_{2}^{\prime}}(A-1)\right| \\
& \times \sum_{i=1}^{A-2} V_{i A-1}\left|\phi_{\alpha_{1}}(1, \ldots, A-2, A-1) \varphi_{\alpha_{2}}(A)\right\rangle
\end{aligned}
$$




$$
\begin{aligned}
= & (A-2)\left\langle\phi_{\alpha_{1}^{\prime}}(1, \ldots, A) \varphi_{\alpha_{2}^{\prime}}(A-1)\left|V_{A-2, A}\right|\right. \\
& \left.\times \phi_{\alpha_{1}}(1, \ldots, A-2, A-1) \varphi_{\alpha_{2}}(A) \mid\right),
\end{aligned}
$$

can be calculated in a similar way to provide the final result

$$
\begin{aligned}
V_{\mathrm{ex}}^{(2)}+V_{\mathrm{ex}}^{(3)}= & (A-2) \sum_{n_{0}^{\prime \prime} n_{0}^{\prime} l_{0}^{\prime} s t}\left(W_{n_{0}^{\prime \prime} n_{0}^{\prime} l_{0}^{\prime} s t}^{\alpha^{\prime}}+W_{n_{0}^{\prime} n_{0}^{\prime \prime} l_{0}^{\prime} s t}^{\alpha^{\prime} \alpha}\right) \\
& \times\left\langle\psi_{n_{0}^{\prime \prime} l_{0}^{\prime}}\left\|V_{A-2, A}^{s t}\right\| \psi_{n_{0}^{\prime} l_{0}^{\prime}}\right\rangle,
\end{aligned}
$$

where

$$
\begin{aligned}
& W_{n_{0}^{\prime \prime} n_{0}^{\prime} l_{0}^{\prime} s t}^{\alpha^{\prime}} \sum_{N^{\prime \prime} \gamma^{\prime \prime} N \Lambda N^{\prime} \Lambda^{\prime} n_{0} l_{0} L_{0} L_{0}^{\prime} S_{0} S_{0}^{\prime} T_{0} T_{0}^{\prime}} T_{L^{\prime \prime}, L_{0} L_{c} \kappa l, L_{0}^{\prime} L_{c}^{\prime} \kappa^{\prime} l^{\prime}, L}^{N \Lambda n_{0} l_{0}, N^{\prime} \Lambda^{\prime} n^{\prime} l_{0}^{\prime}} \\
& \times\left\langle A-1 K_{c} \gamma_{c} \mid A-3 N^{\prime \prime} \gamma^{\prime \prime} ; N \Lambda, n_{0} l_{0} S_{0} T_{0}\left(L_{0}\right): L_{c} S_{c} T_{c}\right\rangle \\
& \times\left\langle A-1 K_{c}^{\prime} \gamma_{c}^{\prime} \mid A-3 N^{\prime \prime} \gamma^{\prime \prime} ; N^{\prime} \Lambda^{\prime}, n_{0}^{\prime \prime} l_{0}^{\prime} s t\left(L_{0}^{\prime}\right): L_{c}^{\prime} S_{c}^{\prime} T_{c}^{\prime}\right\rangle \\
& \times \hat{S}_{c} \hat{S}_{c}^{\prime} \hat{S}_{0} \hat{s} \hat{T}_{c} \hat{T}_{c}^{\prime} \hat{T}_{0} \hat{t}\left\{\begin{array}{ccc}
S & \frac{1}{2} & S_{c}^{\prime} \\
\frac{1}{2} & \frac{1}{2} & s \\
S_{c} & S_{0} & S^{\prime \prime}
\end{array}\right\}\left\{\begin{array}{lll}
T & \frac{1}{2} & T_{c}^{\prime} \\
\frac{1}{2} & \frac{1}{2} & t \\
T_{c} & T_{0} & T^{\prime \prime}
\end{array}\right\} .
\end{aligned}
$$

Finally, the exchange term

$$
\begin{aligned}
V_{\mathrm{ex}}^{(4)}= & \left\langle\phi_{\alpha_{1}^{\prime}}(1, \ldots, A) \varphi_{\alpha_{2}^{\prime}}(A-1)\right| \\
& \times \sum_{i<j}^{A-2} V_{i j}\left|\phi_{\alpha_{1}}(1, \ldots, A-2, A-1), \varphi_{\alpha_{2}}(A)\right\rangle
\end{aligned}
$$

can be caclulated by separating nucleon $A-1$ in the bra vector and nucleon $A$ in the ket vector using one-nucleon fractional parentage expansion and the Talmi-Moshinsky technique. This gives

$$
\begin{aligned}
& V_{\mathrm{ex}}^{(4)}=\sum_{N^{\prime \prime} \gamma^{\prime \prime} \tilde{N}^{\prime \prime} \tilde{\gamma}^{\prime \prime}} \delta_{L^{\prime \prime} \tilde{L}^{\prime \prime}} \delta_{S^{\prime \prime} \tilde{S}^{\prime \prime}} \delta_{T^{\prime \prime} \tilde{T}^{\prime \prime}} U\left(S^{\prime \prime} \frac{1}{2} \frac{1}{2} S ; S_{c} S_{c}^{\prime}\right) \\
& \times(-)^{S+S^{\prime \prime}+S_{c}+S_{c}^{\prime}+T+T^{\prime \prime}+T_{c}+T_{c}^{\prime}} U\left(T^{\prime \prime} \frac{1}{2} \frac{1}{2} T ; T_{c} T_{c}^{\prime}\right) \\
& \times\left\langle A-2 \tilde{N}^{\prime \prime} \tilde{\gamma}^{\prime \prime}\left\|\sum_{i<j}^{A-2} V_{i j}\right\| A-2 N^{\prime \prime} \gamma^{\prime \prime}\right\rangle \\
& \times \sum_{\kappa_{1} l_{1} \kappa_{1}^{\prime} l_{1}^{\prime}}\left\langle A-1 K_{c} \gamma_{c} \mid A-2 N^{\prime \prime} \gamma^{\prime \prime}, \kappa_{1} l_{1}\right\rangle \\
& \times\left\langle A-1 K_{c}^{\prime} \gamma_{c}^{\prime} \mid A-2 N^{\prime \prime} \gamma^{\prime \prime}, \kappa_{1}^{\prime} l_{1}^{\prime}\right\rangle \\
& \times \sum_{L_{0}}(-)^{l^{\prime}+l_{1}^{\prime}-L_{0}} U\left(L^{\prime \prime} l_{1} L 1 ; L_{c} L_{0}\right) U\left(L^{\prime \prime} l_{1}^{\prime} L 1^{\prime} ; L_{c}^{\prime} L_{0}\right) \text {. } \\
& \times\left\langle\kappa^{\prime} l^{\prime} \kappa_{1}^{\prime} l_{1}^{\prime}: L_{0}|A(A-2)| \kappa_{1} l_{1} \kappa l: L_{0}\right\rangle .
\end{aligned}
$$

This expression contains the shell model matrix elements of the potential energy in the $A-2$ core, $\langle A-$ $\left.2 \tilde{N}^{\prime \prime} \tilde{\gamma}^{\prime \prime}\left\|\sum_{i<j}^{A-2} V_{i j}\right\| A-2 N^{\prime \prime} \gamma^{\prime \prime}\right\rangle$, that can be calculated in the usual way.

\section{Overlap integral}

The overlap integral $I(\boldsymbol{r})=\left\langle\Psi_{A-1} \mid \Psi_{A}\right\rangle$ that enters reaction theories, like the distorted wave Born approximation, coupled reaction channels, and breakup and capture theories, is a function of distance between $N$ and the center of mass of $A-1$. It is defined in terms of wave functions $\Psi_{A-1}$ and $\Psi_{A}$ that depend on non-normalized Jacobi coordinates $\boldsymbol{x}_{i}=1 / i \sum_{j=1}^{i} \boldsymbol{r}_{j}-\boldsymbol{r}_{i+1}$. On the contrary, the wave functions in the hyperspherical formalisms can be rewritten in normalized Jacobi coordinates $\boldsymbol{\xi}_{i}=\alpha_{i} \boldsymbol{x}_{i}$, where $\alpha_{i}=\sqrt{i /(i+1)}$. Because

$$
d \rho_{A}=d \xi_{1} \cdots d \xi_{A-1}=\prod_{i=1}^{A-1} \alpha_{i}^{3} d \boldsymbol{x}_{i}
$$

the wave function $\Psi_{A}\left(\left\{\boldsymbol{x}_{i}\right\}\right)$ normalized in coordinates $\left\{\boldsymbol{x}_{i}\right\}$ is related to the wave function $\tilde{\Psi}_{A}\left(\left\{\xi_{i}\right\}\right)$ normalized in coordinates $\left\{\boldsymbol{\xi}_{i}\right\}$ in the following way:

$$
\Psi_{A}\left(\left\{\boldsymbol{x}_{i}\right\}\right)=\left(\prod_{i=1}^{A-1} \alpha_{i}^{3}\right)^{1 / 2} \tilde{\Psi}_{A}\left(\left\{\alpha_{i} \boldsymbol{x}_{i}\right\}\right)=A^{-3 / 4} \tilde{\Psi}_{A}\left(\left\{\alpha_{i} \boldsymbol{x}_{i}\right\}\right) .
$$

Therefore, the overlap integral $I\left(\boldsymbol{x}_{A-1}\right)$ used in reaction theories is related to the overlap integral $\tilde{I}\left(\xi_{A-1}\right)$ obtained in normalized Jacobi coordinates as follows:

$$
\begin{aligned}
I\left(\boldsymbol{x}_{A-1}\right)= & \int d \boldsymbol{x}_{1} \cdots d \boldsymbol{x}_{A-2} \Psi_{A}^{\dagger}\left(\left\{\boldsymbol{x}_{i}\right\}\right) \Psi_{A}\left(\left\{\boldsymbol{x}_{i}\right\}\right) \\
= & \left(\frac{A-1}{A}\right)^{3 / 4} \int d \boldsymbol{\xi}_{1} \cdots d \boldsymbol{\xi}_{A-1} \tilde{\Psi}_{A}^{\dagger}\left(\boldsymbol{\xi}_{1}, \ldots, \boldsymbol{\xi}_{A-2}\right) \\
& \times \tilde{\Psi}_{A}\left(\xi_{1}, \ldots, \boldsymbol{\xi}_{A-2}, \alpha_{A-1} \boldsymbol{x}_{A-1}\right) \\
= & \left(\frac{A-1}{A}\right)^{3 / 4} \tilde{I}\left(\xi_{A-1}\right) .
\end{aligned}
$$

Using the standard for reaction theories partial wave decomposition

$$
\begin{aligned}
& I_{J_{A} J_{A-1}}^{M_{A} M_{A-1}}(\boldsymbol{r})=\sum_{j m_{j} l m \sigma}\left(j m_{j} J_{A-1} M_{A-1} \mid J_{A} M_{A}\right)
\end{aligned}
$$

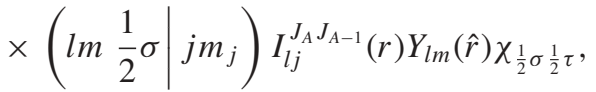

where $\boldsymbol{r} \equiv \boldsymbol{\xi}_{A-1}, J_{i}\left(M_{i}\right)$ is the total momentum (its projection) of nucleus $i, \chi_{\frac{1}{2} \sigma \frac{1}{2} \tau}$ is the spin-isospin function of the separated nucleon with spin (isospin) projection $\sigma(\tau)$, and expanding the ${ }^{5} \mathrm{He}$ wave function in a hyperspherical cluster basis (40), we get the final expression for the radial part of the overlap $\left\langle{ }^{4} \mathrm{He} \otimes n \mid{ }^{5} \mathrm{He}\right\rangle$,

$$
\begin{aligned}
I_{l}(r)= & \left(\frac{4}{5}\right)^{5 / 4} r \sum_{n=0}^{n_{\max }}\langle K=2 n+l \mid K=0, l\rangle \\
& \times \int_{0}^{\infty} d \rho_{c} \frac{\rho_{c}^{4} \chi_{0}^{4} \mathrm{He}\left(\rho_{c}\right) \chi_{n}^{5} \mathrm{He}\left(\sqrt{\rho_{c}^{2}+\frac{4}{5} r^{2}}\right)}{\left(\rho_{c}^{2}+\frac{4}{5} r^{2}\right)^{13 / 4}} \\
& \times P_{n}^{\frac{3}{2}, \frac{7}{2}}\left(\frac{\rho_{c}^{2}+\frac{4}{5} r^{2}}{\rho_{c}^{2}+\frac{4}{5} r^{2}}\right) .
\end{aligned}
$$

Here $\langle K=2 n+l \mid K=0, l\rangle$ is the FPC, which can be calculated via the norm of the hyperspherical cluster harmonics. The overlap $I_{l}(r)$ can be represented as a sum of direct and 
exchange terms. The direct term is an analog to the relative function $g_{l}(r)$ of the traditional microscopic cluster model (46) and it can be obtained by removing the antisymmetrization operator from Eq. (40). The analytical expression for this term is obtained from Eq. (A36) by replacing the FPC $\langle K=2 n+l \mid K=0, l\rangle$ by $5 /\langle K=2 n+l \mid K=0, l\rangle$.
[1] A. Kievsky, S. Rosati, M. Viviani, C. R. Brune, H. J. Karlowski, E. J. Ludwig, and M. H. Wood, Phys. Lett. B406, 292 (1997).

[2] M. Viviani, A. Kievsky, and S. Rosati, Phys. Rev. C 71, 024006 (2005).

[3] N. K. Timofeyuk, Nucl. Phys. A632, 19 (1998).

[4] P. Navratil, C. A. Bertulani, and E. Caurier, Phys. Rev. C 73, 065801 (2006).

[5] K. M. Nollett, R. B. Wiringa, and R. Schiavilla, Phys. Rev. C 63, 024003 (2001).

[6] K. M. Nollett, Phys. Rev. C 63, 054002 (2001).

[7] D. Baye, P.-H. Heenen, and M. Libert-Heinemann, Nucl. Phys. A291, 230 (1977).

[8] P. Descouvemont, Theoretical Models for Nuclear Astrophysics (Nova Science Publishers, Inc., New York, 2003).

[9] A. I. Baz' and M. V. Zhukov, Sov. J. Nucl. Phys. 16, 31 (1973); Sov. J. Nucl. Phys. 16, 529 (1973).

[10] V. D. Efros and M. V. Zhukov, Phys. Lett. B37, 18 (1971); M. V. Zhukov and V. D. Efros, Sov. J. Nucl. Phys. 14, 577 (1972).
[11] L. V. Grigorenko, B. V. Danilin, V. D. Efros, N. B. Shulgina, and M. V. Zhukov, Phys. Rev. C 60, 044312 (1999); 57, 2099 (1998).

[12] B. M. Fisher, C. R. Brune, H. J. Karwowski, D. S. Leonard, E. J. Ludwig, T. C. Black, M. Viviani, A. Kievsky, and S. Rosati, Phys. Rev. C 74, 034001 (2006).

[13] Yu. F. Smirnov and K. V. Shitikova, Sov. J. Part. Nucl. 8, 344 (1977).

[14] N. K. Timofeyuk, Phys. Rev. C 65, 064306 (2002).

[15] I. J. Thompson, B. V. Danilin, V. D. Efros, J. S. Vaagen, J. M. Bang, and M. V. Zhukov, Phys. Rev. C 61, 024318 (2000).

[16] A. B. Volkov, Nucl. Phys. 74, 33 (1965).

[17] V. S. Vasilevsky and G. F. Filippov, Yad. Fiz. 33, 951 (1981).

[18] N. K. Timofeyuk, Phys. Rev. C 69, 034336 (2004).

[19] N. Barnea and A. Novoselsky, Ann. Phys. (NY) 256, 192 (1997).

[20] I. V. Kurdyumov, Yu. F. Smirnov, and K. V. Shitikova, Theor. Math. Fiz. 7, 45 (1971).

[21] L. Trlifaj, Phys. Rev. C 5, 1534 (1972). 